\title{
Tunnel effect for Kramers-Fokker-Planck type operators: return to equilibrium and applications
}

\author{
Frédéric Hérau \\ Laboratoire de Mathématiques \\ Université de Reims \\ Moulin de la Housse B.P. 1039 \\ 51687 Reims cedex 2, France \\ and UMR 6056 CNRS \\ herau@univ-reims.fr \\ Johannes Sjöstrand \\ CMLS \\ Ecole Polytechnique \\ 91128 Palaiseau cedex, France \\ and UMR 7640 CNRS \\ johannes@math.polytechnique.fr
}

Michael Hitrik

Department of Mathematics

University of California

Los Angeles

CA 90095-1555, USA

hitrik@math.ucla.edu

\begin{abstract}
In the first part of this work, we consider second order supersymmetric differential operators in the semiclassical limit, including the Kramers-Fokker-Planck operator, such that the exponent of the associated Maxwellian $\phi$ is a Morse function with two local minima and one saddle point. Under suitable additional assumptions of dynamical nature, we establish the long time convergence to the equilibrium for the associated heat semigroup, with the rate given by the first non-vanishing, exponentially small, eigenvalue. In the second part of the paper, we consider the case when the function $\phi$ has precisely one local minimum and one saddle point. We also discuss further examples of supersymmetric operators, including the Witten Laplacian and the infinitesimal generator for the time evolution of a chain of classical anharmonic oscillators.
\end{abstract}


Keywords and Phrases: Kramers, Fokker-Planck, semiclassical limit, return to equilibrium, heat semigroup, eigenvalue splitting, supersymmetry, Witten Laplacian.

Mathematics Subject Classification 2000: 35P15, 35P20, 47B44, 47D06, 47N55, 81Q60, 82C31

\section{Contents}

1 Introduction and statement of the main result

2 An (hypo-)coercive estimate

3 Supersymmetric operators and return to equilibrium in the double well case $\quad 19$

4 Tunnel effect for a well and the sea 23

5 Some models of KFP type operators 27

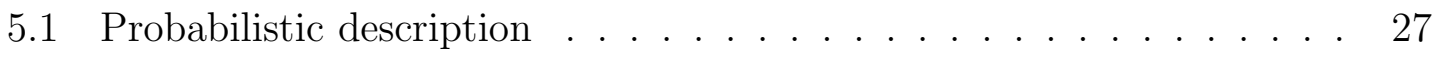

5.2 Witten and Kramers-Fokker-Planck operators . . . . . . . . . . 31

6 Chains of anharmonic oscillators 33

\section{Introduction and statement of the main result}

The principal purpose of the present paper is to apply the spectral results of [12 to obtain a precise information concerning the large time behavior of the heat semigroup generated by the semiclassical Kramers-Fokker-Planck operator

$$
P=y \cdot h \partial_{x}-V^{\prime}(x) \cdot h \partial_{y}+\frac{\gamma}{2}\left(-h \partial_{y}+y\right) \cdot\left(h \partial_{y}+y\right), \quad x, y \in \mathbb{R}^{n}, \quad \gamma>0 .
$$

In fact, as in [12], our main result will be valid for a large class of supersymmetric second order differential operators, including (1.1). Physically, the semiclassical limit $h \rightarrow 0$ in (1.1) corresponds to the régime of low temperatures. Recall that by supersymmetry, we mean the fact that the Kramers-Fokker-Planck operator $P$ can be viewed as a Witten Laplacian in degree 0 associated to a certain non-semidefinite scalar product in the spaces of differential forms. These relations with the Witten complex [22] were exhibited in the works of J. Tailleur, S. Tanase-Nicola, J. Kurchan [21] and J. M. Bismut [1], using respectively the languages of supersymmetry and 
differential forms (See also [8] for a quick introduction to the differential form version of Bismut and [2]).

The paper [12], which is a natural continuation of [11], analyzed resolvent estimates and the low lying eigenvalues of $P$, assuming that the potential $V$ in (1.1) is a smooth real valued Morse function on $\mathbb{R}^{n}$, such that

$$
\partial^{\alpha} V=\mathcal{O}(1), \quad|\alpha| \geq 2,
$$

and with

$$
|\nabla V| \geq 1 / C, \text { for } \quad|x| \geq C>0
$$

Assuming furthermore that $V$ has precisely three critical points: two local minima, $x_{ \pm 1}$, and one critical point $x_{0}$ of index 1, it was established in [12] that for $C>0$ large enough and $h>0$ sufficiently small, the operator $P$ has precisely two eigenvalues in the disc $D(0, h / C)=\left\{z \in \mathbb{C} ;|z|<\frac{h}{C}\right\}, \mu_{0}$ and $\mu_{1}$, such that $\mu_{0}=0$ and $\mu_{1}$ is real and of the form

$$
\mu_{1}=h\left(a_{1}(h) e^{-2 S_{1} / h}+a_{-1}(h) e^{-2 S_{-1} / h}\right), \quad S_{j}=V\left(x_{0}\right)-V\left(x_{j}\right)
$$

Here $a_{j}$ are real with

$$
a_{j}(h) \sim a_{j, 0}+h a_{j, 1}+\ldots \quad a_{j, 0}>0 .
$$

Notice that the eigenfunction corresponding to the eigenvalue $\mu_{0}=0$ is the Maxwellian

$$
\exp (-\phi / h) \in L^{2}\left(\mathbb{R}_{x, y}^{2 n}\right), \quad \phi(x, y)=\frac{y^{2}}{2}+V(x) .
$$

In the case when $V \rightarrow+\infty$ as $x \rightarrow \infty$ and $V$ has precisely one local minimum, it follows from the results of [11] that in a disc $D(0, C h), C \geq 1$, apart from the eigenvalue $\mu_{0}=0$, the real part of the other eigenvalues is $\geq h / C$. In this case, precise results describing the behavior of the semigroup $\exp (-t P / h)$ for large $t$, were obtained in [11] - the rate of the return to equilibrium, given by the projection onto the ground state (1.6), is then of the order of magnitude 1. In this work, we shall complement this study by analyzing the question of a return to equilibrium in the presence of exponentially small eigenvalues, due to the tunneling between the local minima.

The following is the main result of this work, specialized to the case of the Kramers-Fokker-Planck operator (1.1). Here we shall also write $P$ for the m-accretive realization of the operator (1.1) - see also section 2 and section 3 in [12]. 
Theorem 1.1 Assume that $V$ in (1.1) is a $C^{\infty}$ real valued Morse function satisfying (1.2) and (1.3) and having precisely 3 critical points: 2 local minima, $x_{ \pm 1}$, and a critical point of index 1 , so that the disc $D(0, h / C)$ for $C>0$ large enough, contains precisely 2 eigenvalues of $P, \mu_{0}=0$ and $\mu_{1}$ given in (1.4). Let $\Pi_{j}$ be the spectral projection associated with the eigenvalue $\mu_{j}, j=0,1$. Then we have

$$
\Pi_{j}=\mathcal{O}(1), \quad h \rightarrow 0 .
$$

We have furthermore, uniformly as $t \geq 0$ and $h \rightarrow 0$,

$$
e^{-t P / h}=\Pi_{0}+e^{-t \mu_{1} / h} \Pi_{1}+\mathcal{O}(1) e^{-t / C}, \quad C>0, \quad \text { in } \quad \mathcal{L}\left(L^{2}, L^{2}\right) .
$$

The structure of the article is as follows: In section 2, relying upon the results of [12], [11], we establish the basic a priori coercivity estimate for the operator $P$ in a suitable exponentially weighted space, introduced in [12]. In this sense it can be interpreted as an hypocoercive estimate (see e.g. [13], [23]). In section 3 it is then quite straightforward to prove Theorem 1.1 in its general form, by combining the results of section 2 together with the analysis of [12].

The second part of the paper, consisting of sections 4-6 is of a somewhat different nature, complementing and extending the previous analysis. In section 4, we study the case left out in [12], when the potential $V$ in (1.1) has precisely two critical points: one local minimum and a critical point of index one. In this case, 0 is not an eigenvalue of $P$, and the large time behavior of the heat semigroup is governed entirely by the first, exponentially small, eigenvalue. In section 5, we give some examples and describe a probabilistic framework in which the Witten Laplacian and the Kramers-Fokker-Planck operator both arise naturally. Finally, in section 6, we describe another example of a supersymmetric operator, for which the question of a convergence to equilibrium is of interest, namely a chain of classical interacting anharmonic oscillators, coupled to a heat bath. We show how to adapt the analysis of 12 to cover also this case.

Acknowledgments: A part of this work was carried out in May of 2007, when the second author was visiting Université Paris 13 being on leave from UCLA. It is a great pleasure for him to thank its Département de Mathématiques, and in particular Alain Grigis, for the extraordinary hospitality and excellent working conditions. His best thanks are also due to the Centre de Mathématiques of École Polytechnique for the generous hospitality in June of 2007. The partial support of his research by the National Science Foundation under grant DMS-0653275 and by an Alfred P. Sloan Research Fellowship is also gratefully acknowledged. 


\section{An (hypo-)coercive estimate}

The purpose of this section is to establish an a priori estimate for $P$, instrumental in proving Theorem 1.1. This estimate will imply the exponential decay for the heat semigroup $\exp (-t P / h)$ in $L^{2}$, when restricted to the kernel of the spectral projection corresponding to the eigenvalues $\mu_{0}=0$ and $\mu_{1}$ in (1.4). When doing so, as in [12, rather than working directly with (1.1), we shall consider a broader class of scalar real second order non-elliptic non-selfadjoint operators on $\mathbb{R}^{n}$. For completeness, we shall now recall, following [12], the definition and the main assumptions concerning this class.

Let us consider

$$
\begin{aligned}
P & =\sum_{j, k=1}^{n} h D_{x_{j}} \circ b_{j, k}(x) \circ h D_{x_{k}}+\frac{1}{2} \sum_{j=1}^{n}\left(c_{j}(x) h \partial_{x_{j}}+h \partial_{x_{j}} \circ c_{j}(x)\right)+p_{0}(x) \\
& =: \quad P_{2}+i P_{1}+P_{0}, \quad D_{x_{j}}=\frac{1}{i} \frac{\partial}{\partial_{x_{j}}} .
\end{aligned}
$$

Here the coefficients $b_{j, k}, c_{j}, p_{0}$ all belong to $C^{\infty}\left(\mathbb{R}^{n} ; \mathbb{R}\right)$, with $b_{j, k}=b_{k, j}$. Associated to $P$ in (2.1) is the symbol in the semiclassical sense,

$$
\begin{gathered}
p(x, \xi)=p_{2}(x, \xi)+i p_{1}(x, \xi)+p_{0}(x), \\
p_{2}(x, \xi)=\sum_{j, k=1}^{n} b_{j, k}(x) \xi_{j} \xi_{k}, \quad p_{1}(x, \xi)=\sum_{j=1}^{n} c_{j}(x) \xi_{j},
\end{gathered}
$$

so that $p_{j}(x, \xi)$ is a real-valued polynomial in $\xi$, positively homogeneous of degree $j$, $0 \leq j \leq 2$. We may notice that $p(x, \xi)$ coincides with the Weyl symbol of $P$ modulo $\mathcal{O}\left(h^{2}\right)$, locally uniformly.

As in [12], we shall assume that

$$
p_{2}(x, \xi) \geq 0, \quad p_{0}(x) \geq 0 .
$$

Furthermore, we shall impose the following growth conditions,

$$
\begin{aligned}
\partial_{x}^{\alpha} b_{j, k}(x)=\mathcal{O}(1), & |\alpha| \geq 0, \\
\partial_{x}^{\alpha} c_{j}(x)=\mathcal{O}(1), & |\alpha| \geq 1, \\
\partial_{x}^{\alpha} p_{0}(x)=\mathcal{O}(1), \quad & |\alpha| \geq 2 .
\end{aligned}
$$

From section 3 in [12], we recall that under these assumptions, the graph closure of $P: \mathcal{S}\left(\mathbb{R}^{n}\right) \rightarrow \mathcal{S}\left(\mathbb{R}^{n}\right)$, still denoted by $P$ and such that $\operatorname{Re} P \geq 0$, coincides with the 
maximal closed realization of $P$, with the domain given by $\mathcal{D}(P)=\left\{u \in L^{2} ; P u \in\right.$ $\left.L^{2}\right\}$. In particular, this shows that the operator $P$ is m-accretive, and hence, the contraction semigroup

$$
e^{-t P / h}: L^{2} \rightarrow L^{2}, \quad t \geq 0
$$

is well-defined.

We proceed next to recall the additional assumptions of a dynamical nature, introduced in section 4 of [12]. Let

$$
\nu\left(x, \partial_{x}\right)=\sum_{j=1}^{n} c_{j}(x) \partial_{x_{j}},
$$

and recall the Hypothesis 4.1 of [12]:

The set $\left\{x \in \mathbb{R}^{n} ; p_{0}(x)=0, \nu\left(x, \partial_{x}\right)=0\right\}$ is finite $=\left\{x_{1}, \ldots x_{N}\right\}$.

With $\rho_{j}=\left(x_{j}, 0\right), 1 \leq j \leq N$, we define the critical set

$$
\mathcal{C}=\left\{\rho_{1}, \ldots \rho_{N}\right\} \subset \mathbb{R}^{2 n} .
$$

The coefficients $p_{0}, p_{1}, p_{2}$ in (2.2) all vanish to the second order at each $\rho_{j} \in \mathcal{C}$. As in [12], we define

$$
\widetilde{p}(x, \xi)=p_{0}(x)+\langle\xi\rangle^{-2} p_{2}(x, \xi),
$$

and consider the time average

$$
\langle\widetilde{p}\rangle_{T_{0}}=\frac{1}{T_{0}} \int_{-T_{0} / 2}^{T_{0} / 2} \tilde{p} \circ \exp \left(t H_{p_{1}}\right) d t, \quad T_{0}>0 .
$$

We shall assume that the Hypothesis 4.3 of [12] holds true:

$$
\text { For } T_{0}>0 \text { fixed, we have near each } \rho_{j},\langle\widetilde{p}\rangle_{T_{0}}(\rho) \sim\left|\rho-\rho_{j}\right|^{2},
$$

and in any set of the form $|x| \leq C$, $\operatorname{dist}(\rho, \mathcal{C}) \geq 1 / C$, we have

$$
\langle\widetilde{p}\rangle_{T_{0}}(\rho) \geq \frac{1}{\widetilde{C}(C)}, \quad \widetilde{C}(C)>0 .
$$

We also need an additional dynamical hypothesis near $\infty$ in $\mathbb{R}^{n}$,

$$
\begin{aligned}
& \forall \text { neighborhood } U \text { of } \pi_{x} \mathcal{C}, \text { and } \forall x \in \mathbb{R}^{n} \backslash U, \exists C>0, \\
& \text { meas }\left(\left\{t \in\left[-\frac{T_{0}}{2}, \frac{T_{0}}{2}\right] ; p_{0}(\exp t \nu(x)) \geq \frac{1}{C}\right\}\right) \geq \frac{1}{C} .
\end{aligned}
$$


Under the assumptions above, the paper [12] defines an auxiliary real valued weight function $\psi_{\varepsilon}(x, \xi)=\mathcal{O}(\varepsilon)$ on $T^{*} \mathbb{R}^{n}, \varepsilon>0$, such that

$$
\partial_{x}^{\alpha} \partial_{\xi}^{\beta} \psi_{\varepsilon}(x, \xi)=\mathcal{O}\left(\varepsilon^{1-|\alpha+\beta| / 2}\langle\xi\rangle^{-|\beta|}\right)
$$

together with an associated canonical transformation

$$
\kappa(\delta): \mathbb{R}^{2 n} \rightarrow \Lambda_{\delta}:=\left\{(x, \xi)+i \delta H_{\psi_{\varepsilon}}(x, \xi) ;(x, \xi) \in \mathbb{R}^{2 n}\right\}, \quad 0<\delta \ll 1,
$$

for which

$$
\kappa(\delta)(x, \xi)=(x, \xi)+i \delta H_{\psi_{\varepsilon}}(x, \xi)+\mathcal{O}\left(\varepsilon^{1 / 2} \delta^{2}\right) .
$$

Here we let $H_{f}$ denote the Hamilton field $f_{\xi}^{\prime}(x, \xi) \cdot \partial_{x}-f_{x}^{\prime}(x, \xi) \cdot \partial_{\xi}$ of a $C^{1}$-function $f(x, \xi)$. We refer to section 4 of [12] for the details of the construction of $\psi_{\varepsilon}$ and $\kappa_{\delta}$. Here we shall merely recall that $\delta>0$ fixed in (2.18) should be small enough, and $\varepsilon=A h$, with $A$ arbitrarily large but fixed.

Associated to $\kappa(\delta)$ in (2.17) there is an elliptic Fourier integral operator with a complex phase $A_{\delta, \varepsilon}$, constructed in Section 5 of [12], such that

$$
A_{\delta, \varepsilon}: \mathcal{S} \rightarrow \mathcal{S}
$$

continuously, and

$$
A_{\delta, \varepsilon}=\mathcal{O}_{A}(1): L^{2} \rightarrow L^{2}
$$

Moreover, it is proved in [12] that $A_{\delta, \varepsilon}$ is invertible, when $\varepsilon / h \gg 1$, with the inverse $A_{\delta, \varepsilon}^{-1}$ also enjoying the mapping properties (2.19), (2.20).

When $B \geq A$ fixed is to be chosen, and $\widetilde{A} \gg B$ is large enough, as in sections 6,7 of [12], we shall consider the conjugated operator

$$
P^{\delta, \widetilde{\varepsilon}}=A_{\delta, \widetilde{\varepsilon}}^{-1} P A_{\delta, \widetilde{\varepsilon}}, \quad \widetilde{\varepsilon}=\widetilde{A} h,
$$

acting on $L^{2}\left(\mathbb{R}^{n}\right)$. It was then proved in $\left[12\right.$ that the real part of the symbol of $P^{\delta, \widetilde{\varepsilon}}$ is $\geq \frac{\delta \widetilde{\varepsilon}}{C}-C h$ outside of $\mathcal{C}+B(0, \sqrt{\widetilde{\varepsilon}}), C>0$. In the set $\mathcal{C}+B(0, \sqrt{\widetilde{\varepsilon}})$, the symbol of $P^{\delta, \widetilde{\varepsilon}}$ is independent of $\widetilde{\varepsilon}$ modulo $\mathcal{O}\left(\widetilde{\varepsilon}\left(\frac{h}{\widetilde{\varepsilon}}\right)^{\infty}\right)$ and is of the form

$$
\widetilde{P}^{\delta} \sim p^{\delta}+h^{2} r_{2}+\ldots
$$

where

$$
\left|p^{\delta}(\rho)\right| \sim \operatorname{dist}(\rho, \mathcal{C})^{2}, \quad \operatorname{Re} p^{\delta}(\rho) \sim \operatorname{dist}(\rho, \mathcal{C})^{2} .
$$

Here we write $B(0, r)=\left\{\rho \in \mathbb{R}^{2 n} ;|\rho|<r\right\}, r>0$. 
As has also been recalled in section 8 in [12], in the set $\mathcal{C}+B(0, \sqrt{\widetilde{\varepsilon}})$, we have

$$
P^{\delta, \widetilde{\varepsilon}}-\widetilde{P}^{\delta}=\widetilde{\mathcal{O}}\left(\widetilde{\varepsilon}\left(\frac{h}{\widetilde{\varepsilon}}\right)^{\infty}\right)
$$

while when away from $\mathcal{C}+B(0, \sqrt{\widetilde{\varepsilon}})$, we shall use that

$$
P^{\delta, \widetilde{\varepsilon}}-\widetilde{P}^{\delta}=\widetilde{\mathcal{O}}\left(\widetilde{\varepsilon}+\rho^{2}\right)
$$

Here, following [12], we use the notation $f_{\varepsilon}=\widetilde{\mathcal{O}}(\varepsilon)$ to express that

$$
\partial_{x}^{\alpha} \partial_{\xi}^{\beta} f_{\varepsilon}(x, \xi)=\mathcal{O}\left(\varepsilon^{1-|\alpha+\beta| / 2}\langle\xi\rangle^{-|\beta|}\right)
$$

for arbitrary multi-indices $\alpha, \beta \in \mathbb{N}^{n}$.

We shall study estimates for the real part of the quadratic form associated to the operator $P^{\delta, \widetilde{\varepsilon}}$. The starting point here is Proposition 7.1 of [12]: let $0 \leq k_{\varepsilon}=\widetilde{\mathcal{O}}(\varepsilon)$ be equal to $\varepsilon$ in $\mathcal{C}+B(0, \sqrt{\varepsilon})$ and have its support in $\mathcal{C}+B(0, \sqrt{2 \varepsilon})$. Let

$$
K_{\varepsilon}=\mathrm{Op}_{h}\left(k_{\varepsilon}\right)
$$

stand for the Weyl quantization of $k_{\varepsilon}(x, h \xi)$. It is then established in Proposition 7.1 of [12] that

$$
\operatorname{Re}\left(\left(P^{\delta, \varepsilon}+K_{\varepsilon}\right) u \mid u\right) \geq\left(\frac{\delta \varepsilon}{C}-C h\right)\|u\|^{2}, \quad u \in \mathcal{S},
$$

when $\varepsilon=A h, C>0$ is independent of $\delta, A$, and $h$ is small enough depending on these 2 parameters.

Rather than working with the estimate (2.25), we shall use that

$$
\operatorname{Re}\left(\left(P^{\delta, \widetilde{\varepsilon}}+K_{\varepsilon}\right) u \mid u\right) \geq\left(\frac{\delta \varepsilon}{C}-C h\right)\|u\|^{2}, \quad u \in \mathcal{S},
$$

which is proved in exactly the same way as in section 7 of [12]. Here we recall that $\varepsilon=A h, \widetilde{\varepsilon}=\widetilde{A} h, \widetilde{A} \gg A$. In what follows we shall use that the estimate (2.26) holds also for $u \in \mathcal{D}\left(P^{\delta, \widetilde{\varepsilon}}\right)=\left\{u \in L^{2} ; P^{\delta, \widetilde{\varepsilon}} u \in L^{2}\right\}=A_{\delta, \widetilde{\varepsilon}}^{-1} \mathcal{D}(P)$.

Let

$$
\Pi_{B}=\Pi_{B}^{\delta, \widetilde{\varepsilon}}
$$


be the spectral projection of $P^{\delta, \widetilde{\varepsilon}}$ associated with the spectrum of $P^{\delta, \widetilde{\varepsilon}}$ in the open disc $D(0, B h)$. From Theorem 8.3 in [12] we recall that the spectrum of $P^{\delta, \widetilde{\varepsilon}}$ in $D(0, B h)$ is discrete, and the eigenvalues are of the form

$$
\lambda_{j, k}(h) \sim h\left(\mu_{j, k}+h^{1 / N_{j, k}} \mu_{j, k, 1}+h^{2 / N_{j, k}} \mu_{j, k, 2}+\ldots\right)
$$

where the $\mu_{j, k}$ are all numbers in $D(0, B)$ of the form

$$
\mu_{j, k}=\frac{1}{i} \sum_{\ell=1}^{n}\left(\nu_{j, k, \ell}+\frac{1}{2}\right) \lambda_{j, \ell}, \quad \nu_{j, k, \ell} \in \mathbb{N}
$$

for some $j \in\{1, \ldots N\}$. Here $\lambda_{j, \ell}, 1 \leq \ell \leq n$, are the eigenvalues of the Hamilton map of the quadratic part of $p$ at $\rho_{j} \in \mathcal{C}$, for which $\operatorname{Im} \lambda_{j, \ell}>0$. Here we also assume that $B$ is chosen such that $\left|\mu_{j, k}\right| \neq B$, for all $j, k$.

Assume that $u \in L^{2}$ is such that

$$
u \in \operatorname{Ran}\left(1-\Pi_{B}\right)
$$

We are interested in lower bounds for

$$
\operatorname{Re}\left(P^{\delta, \widetilde{\varepsilon}} u \mid u\right)
$$

which, in view of (2.26), amounts to estimating $K_{\varepsilon} u$. In doing so, we shall assume, for notational simplicity only, that the critical set $\mathcal{C}$ defined in (2.11) consists of a single point, $\rho_{1}=(0,0)$. From (2.23), we know that the leading symbol of $\widetilde{P}^{\delta}, p^{\delta}$, is such that

$$
\left|p^{\delta}(\rho)\right| \sim|\rho|^{2}, \quad \rho \in B(0, \sqrt{\widetilde{\varepsilon}}) .
$$

Let

$$
p_{0}(x, \xi)=p_{0}^{\delta}(x, \xi)=\sum_{|\alpha+\beta|=2} \frac{\partial_{x}^{\alpha} \partial_{\xi}^{\beta} p^{\delta}(0,0)}{\alpha ! \beta !} x^{\alpha} \xi^{\beta}
$$

be the quadratic approximation of $p^{\delta}$, so that

$$
p^{\delta}-p_{0}=\mathcal{O}\left((x, \xi)^{3}\right)=\mathcal{O}\left(\left(h+(x, \xi)^{2}\right)^{3 / 2}\right), \quad(x, \xi) \rightarrow(0,0) .
$$

Then $p_{0}$ is an elliptic quadratic form on $\mathbb{R}^{2 n}$, with a positive definite real part. The quadratic differential operator

$$
P_{0}=\mathrm{Op}_{h}\left(p_{0}\right): L^{2} \rightarrow L^{2},
$$


has discrete spectrum, and from [20] we know that the eigenvalues of $P_{0}$ are of the form $h \mu_{1, k}$, with $\mu_{1, k}$ defined as in (2.27).

When estimating $K_{\varepsilon} u$ for $u \in \operatorname{Ran}\left(1-\Pi_{B}\right)$, we also introduce the spectral projection $\Pi_{0, B}$ associated to $P_{0}$ and the spectrum of $P_{0}$ in $D(0, B h)$. Then, since $\Pi_{B} u=0$,

$$
K_{\varepsilon} u=K_{\varepsilon}\left(\Pi_{0, B}-\Pi_{B}\right) u+K_{\varepsilon}\left(1-\Pi_{0, B}\right) u .
$$

We shall estimate the first term in the right hand side of (2.31), using the following result.

Lemma 2.1 We have

$$
\Pi_{B}-\Pi_{0, B}=\mathcal{O}_{B}\left(\widetilde{A}^{3 / 2} h^{1 / 2}+\widetilde{A}^{-1}\right): L^{2} \rightarrow L^{2}
$$

Proof: Let $\chi \in C_{0}^{\infty}(B(0,2)), 0 \leq \chi \leq 1$, be such that $\chi(x, \xi)=1$ for $|(x, \xi)| \leq 1$. Set $\chi_{\sqrt{\widetilde{\varepsilon}}}(x, \xi)=\chi\left(\widetilde{\varepsilon}^{-1 / 2}(x, \xi)\right)$. We shall first show that

$$
\Pi_{0, B}\left(1-\chi_{\sqrt{\widetilde{\varepsilon}}}\right)=\mathcal{O}_{B}\left(\left(\frac{h}{\widetilde{\varepsilon}}\right)^{\infty}\right): L^{2} \rightarrow L^{2}
$$

and similarly, that

$$
\Pi_{B}\left(1-\chi_{\sqrt{\widetilde{\varepsilon}}}\right)=\mathcal{O}_{B}\left(\left(\frac{h}{\widetilde{\varepsilon}}\right)^{\infty}\right): L^{2} \rightarrow L^{2} .
$$

When proving (2.33), we shall use the well-posed Grushin problem for the quadratic operator $P_{0}$, described in [11], [12]. Let

$$
\Lambda=\left\langle\frac{(x, h D)}{h^{1 / 2}}\right\rangle=\left(1+\frac{x^{2}+\left(h D_{x}\right)^{2}}{h}\right)^{1 / 2},
$$

so that the quadratic elliptic operator $P_{0}$ is equipped with the natural domain

$$
\mathcal{D}\left(P_{0}\right)=\left\{u \in L^{2} ; \Lambda^{2} u \in L^{2}\right\}
$$

In section 11 in [11], using the generalized eigenfunctions of $P_{0}$ and of the adjoint $P_{0}^{*}$, the authors have constructed the operators

$$
R_{-}: \mathbb{C}^{N_{0}} \rightarrow L^{2}, \quad R_{+}: L^{2} \rightarrow \mathbb{C}^{N_{0}}, \quad N_{0} \in \mathbb{N}
$$


such that when $z \in D(0, B h)$, the problem

$$
\left(P_{0}-z\right) u+R_{-} u_{-}=v, \quad R_{+} u=v_{+},
$$

for $v \in L^{2}, v_{+} \in \mathbb{C}^{N_{0}}$ has a unique solution $u \in \mathcal{D}\left(P_{0}\right), u_{-} \in \mathbb{C}^{N_{0}}$. Moreover, we have the a priori estimate

$$
h\left\|\Lambda^{2} u\right\|+\left|u_{-}\right| \leq \mathcal{O}(1)\left(\|v\|+h\left|v_{+}\right|\right) .
$$

Associated to (2.37) is the Grushin operator

$$
\mathcal{P}_{0}(z)=\left(\begin{array}{cc}
P_{0}-z & R_{-} \\
R_{+} & 0
\end{array}\right): \mathcal{D}(P) \times \mathbb{C}^{N_{0}} \rightarrow L^{2} \times \mathbb{C}^{N_{0}}, \quad z \in D(0, B h),
$$

with an inverse

$$
\mathcal{E}_{0}(z)=\left(\begin{array}{ll}
E(z) & E_{+}(z) \\
E_{-}(z) & E_{-+}(z)
\end{array}\right): L^{2} \times \mathbb{C}^{N_{0}} \rightarrow \mathcal{D}(P) \times \mathbb{C}^{N_{0}},
$$

depending holomorphically on $z$. From section 11 of [11], we recall that $\mathcal{E}_{0}(z)$ enjoys the following localization properties, when $k \in \mathbb{R}$,

$$
\Lambda^{2-k} E(z) \Lambda^{k}=\mathcal{O}\left(\frac{1}{h}\right): L^{2} \rightarrow L^{2}
$$

and

$$
\Lambda^{k} E_{+}(z)=\mathcal{O}_{k}(1): \mathbb{C}^{N_{0}} \rightarrow L^{2}, \quad E_{-}(z) \Lambda^{k}=\mathcal{O}_{k}(1): L^{2} \rightarrow \mathbb{C}^{N_{0}},
$$

Let $\gamma \subset D(0, B)$ be a simple positively oriented closed $h$-independent contour, such that all eigenvalues of $P_{0}$ and $P^{\delta, \widetilde{\varepsilon}}$ in $D(0, B h)$ are contained in the interior of $h \gamma$, so that we have

$$
\operatorname{dist}\left(z, \operatorname{Spec}\left(P_{0}\right) \cup \operatorname{Spec}\left(P^{\delta, \widetilde{\varepsilon}}\right)\right) \geq h / \mathcal{O}(1), \quad z \in h \gamma .
$$

Here we continue to assume that $B>0$ is chosen so that there are no numbers of the form $\mu_{j, k}$ in (2.27) on the boundary of $D(0, B)$. Writing

$$
\Pi_{0, B}=\frac{1}{2 \pi i} \int_{h \gamma}\left(z-P_{0}\right)^{-1} d z
$$

and using the well-known formula

$$
\left(z-P_{0}\right)^{-1}=-E(z)+E_{+}(z) E_{-+}(z)^{-1} E_{-}(z),
$$


we obtain that

$$
\Pi_{0, B}=\frac{1}{2 \pi i} \int_{h \gamma} E_{+}(z) E_{-+}(z)^{-1} E_{-}(z) d z .
$$

Now (2.42) gives that for each $k \in \mathbb{N}$,

$$
E_{-}(z)\left(1-\chi_{\sqrt{\widetilde{\varepsilon}}}\right)=E_{-} \Lambda^{k} \Lambda^{-k}\left(1-\chi_{\sqrt{\widetilde{\varepsilon}}}\right)=\mathcal{O}\left(\left(\frac{h}{\widetilde{\varepsilon}}\right)^{k / 2}\right): L^{2} \rightarrow \mathbb{C}^{N_{0}} .
$$

Using also that along $h \gamma$, we have

$$
E_{+}(z)=\mathcal{O}(1): \mathbb{C}^{N_{0}} \rightarrow L^{2}
$$

and

$$
E_{-+}(z)^{-1}=\mathcal{O}\left(h^{-1}\right): \mathbb{C}^{N_{0}} \rightarrow \mathbb{C}^{N_{0}},
$$

as well as the fact that length of $h \gamma$ is $\mathcal{O}_{B}(h)$, we obtain (2.33).

The proof of (2.34) proceeds along the similar lines, relying upon the well-posed Grushin problem for $P^{\delta, \widetilde{\varepsilon}}-z$, constructed from the Grushin problem for $P_{0}$ and described in detail in section 11 of [11] and section 8 of [12]. In particular, the analogue of the localization property (2.42) holds true for the inverse of the Grushin operator for $P^{\delta, \widetilde{\varepsilon}}$, and arguing as above, we get (2.34).

We now come to consider estimates for the difference $\left(\Pi_{B}-\Pi_{0, B}\right) \chi_{\sqrt{\widetilde{\varepsilon}}}$, where we claim that

$$
\left(\Pi_{B}-\Pi_{0, B}\right) \chi_{\sqrt{\widetilde{\varepsilon}}}=\mathcal{O}_{B}\left(\frac{\widetilde{\varepsilon}^{3 / 2}}{h}+\frac{1}{\widetilde{A}}\right): L^{2} \rightarrow L^{2}, \quad \widetilde{\varepsilon}=\widetilde{A} h .
$$

In view of (2.33) and (2.34), this will complete the proof of Lemma 2.1.

When proving (2.47), we shall first estimate the difference $\left(\widetilde{\Pi}_{B}-\Pi_{0, B}\right) \chi_{\sqrt{\widetilde{\varepsilon}}}$, where $\widetilde{\Pi}_{B}$ is the spectral projection of the operator $\widetilde{P}^{\delta}$ associated with the spectrum of $\widetilde{P}^{\delta}$ in $D(0, B h)$. Using 2.43) together with the similar formula for $\widetilde{\Pi}_{B}$, we get, by an application of the resolvent identity,

$$
\left(\widetilde{\Pi}_{B}-\Pi_{0, B}\right) \chi_{\sqrt{\widetilde{\varepsilon}}}=\frac{1}{2 \pi i} \int_{h \gamma}\left(z-\widetilde{P}^{\delta}\right)^{-1}\left(\widetilde{P}^{\delta}-P_{0}\right)\left(z-P_{0}\right)^{-1} \chi_{\sqrt{\widetilde{\varepsilon}}} d z .
$$

Now (2.41) (2.42), (2.44), together with the fact that along $h \gamma$ we have $\left\|E_{-+}^{-1}(z)\right\|=$ $\mathcal{O}\left(h^{-1}\right)$, imply that for $k \in \mathbb{R}$,

$$
\Lambda^{k}\left(z-P_{0}\right)^{-1} \Lambda^{-k}=\mathcal{O}\left(\frac{1}{h}\right): L^{2} \rightarrow L^{2},
$$


and even that

$$
\Lambda^{2+k}\left(z-P_{0}\right)^{-1} \Lambda^{-k}=\mathcal{O}\left(\frac{1}{h}\right): L^{2} \rightarrow L^{2} .
$$

Here we shall take $k=3$ in (2.49). Writing the integrand in (2.48) as

$$
\begin{aligned}
& \left(z-\widetilde{P}^{\delta}\right)^{-1}\left(\widetilde{P}^{\delta}-P_{0}\right)\left(z-P_{0}\right)^{-1} \chi_{\sqrt{\widetilde{\varepsilon}}} \\
= & \left(z-\widetilde{P}^{\delta}\right)^{-1}\left(\widetilde{P}^{\delta}-P_{0}\right) \Lambda^{-3} \Lambda^{3}\left(z-P_{0}\right)^{-1} \Lambda^{-3} \Lambda^{3} \chi_{\sqrt{\widetilde{\varepsilon}}}
\end{aligned}
$$

we see that we have to estimate the operator norm of $\left(\widetilde{P}^{\delta}-P_{0}\right) \Lambda^{-3}$. Now it follows from (2.22), (2.30) that

$$
\left(\widetilde{P}^{\delta}-P_{0}\right) \Lambda^{-3}=\mathcal{O}\left(h^{3 / 2}\right): L^{2} \rightarrow L^{2} .
$$

Also,

$$
\Lambda^{3} \chi_{\sqrt{\tilde{\varepsilon}}}=\mathcal{O}\left(\frac{\widetilde{\varepsilon}^{3 / 2}}{h^{3 / 2}}\right): L^{2} \rightarrow L^{2} .
$$

Combining (2.48), (2.49), (2.52), (2.53) together with the fact that

$$
\left(z-\widetilde{P}^{\delta}\right)^{-1}=\mathcal{O}\left(h^{-1}\right): L^{2} \rightarrow L^{2}, \quad z \in h \gamma,
$$

which follows from Theorem 8.4 in [12], and that the length of $h \gamma$ is $\mathcal{O}_{B}(h)$, we obtain that

$$
\left(\widetilde{\Pi}_{B}-\Pi_{0, B}\right) \chi_{\sqrt{\widetilde{\varepsilon}}}=\mathcal{O}_{B}\left(\frac{\widetilde{\varepsilon}^{3 / 2}}{h}\right): L^{2} \rightarrow L^{2}
$$

It only remains now to estimate the operator norm of $\left(\Pi_{B}-\widetilde{\Pi}_{B}\right) \chi_{\sqrt{\varepsilon}}$. To that end, we write, as in (2.48),

$$
\begin{aligned}
\left(\Pi_{B}-\widetilde{\Pi}_{B}\right) \chi_{\sqrt{\widetilde{\varepsilon}}}= & \frac{1}{2 \pi i} \int_{h \gamma}\left(z-P^{\delta, \widetilde{\varepsilon}}\right)^{-1}\left(P^{\delta, \widetilde{\varepsilon}}-\widetilde{P}^{\delta}\right)\left(z-\widetilde{P}^{\delta}\right)^{-1} \chi_{\sqrt{\widetilde{\varepsilon}}} d z \\
= & \frac{1}{2 \pi i} \int_{h \gamma}\left(z-P^{\delta, \widetilde{\varepsilon}}\right)^{-1}\left(P^{\delta, \widetilde{\varepsilon}}-\widetilde{P}^{\delta}\right) \chi_{\sqrt{\varepsilon}}\left(z-\widetilde{P}^{\delta}\right)^{-1} \chi_{\sqrt{\tilde{\varepsilon}}} d z \\
& +\frac{1}{2 \pi i} \int_{h \gamma}\left(z-P^{\delta, \widetilde{\varepsilon}}\right)^{-1}\left(P^{\delta, \widetilde{\varepsilon}}-\widetilde{P}^{\delta}\right)\left(1-\chi_{\sqrt{\widetilde{\varepsilon}}}\right)\left(z-\widetilde{P}^{\delta}\right)^{-1} \chi_{\sqrt{\widetilde{\varepsilon}}} d z \\
= & \mathrm{I}+\mathrm{II},
\end{aligned}
$$


with the natural definitions of I and II. Using, as in section 8 of [12], that

$$
\left(P^{\delta, \widetilde{\varepsilon}}-\widetilde{P}^{\delta}\right) \chi_{\sqrt{\widetilde{\varepsilon}}}=\mathcal{O}\left(h \frac{h}{\widetilde{\varepsilon}}\right): L^{2} \rightarrow L^{2},
$$

together with the $\mathcal{O}\left(h^{-1}\right)$-estimates for the resolvents of $P^{\delta, \widetilde{\varepsilon}}$ and $\widetilde{P}^{\delta}$ along the contour $h \gamma$, we get

$$
\mathrm{I}=\mathcal{O}_{B}\left(\frac{1}{h} \widetilde{\varepsilon}\right)=\mathcal{O}_{B}\left(\frac{1}{\widetilde{A}}\right): L^{2} \rightarrow L^{2} .
$$

We now come to estimate the term II in (2.55). We have

$$
\chi_{\sqrt{\widetilde{\varepsilon}}}\left(P^{\delta, \widetilde{\varepsilon}}-\widetilde{P}^{\delta}\right)=\mathcal{O}\left(h \frac{h}{\widetilde{\varepsilon}}\right): L^{2} \rightarrow L^{2},
$$

and using this estimate, as well as the $\mathcal{O}\left(h^{-1}\right)$-resolvent bounds for $P^{\delta, \widetilde{\varepsilon}}$ and $\widetilde{P}^{\delta}$, we see that modulo a term whose operator norm on $L^{2}$ is

$$
\mathcal{O}_{B}\left(\frac{h}{\widetilde{\varepsilon}}\right)=\mathcal{O}_{B}\left(\widetilde{A}^{-1}\right),
$$

we may replace the integrand in II by the following expression,

$$
\left(z-P^{\delta, \widetilde{\varepsilon}}\right)^{-1}\left(1-\chi_{\sqrt{\widetilde{\varepsilon}}}\right)\left(P^{\delta, \widetilde{\varepsilon}}-\widetilde{P}^{\delta}\right)\left(1-\chi_{\sqrt{\widetilde{\varepsilon}}}\right)\left(z-\widetilde{P}^{\delta}\right)^{-1} \chi_{\sqrt{\widetilde{\varepsilon}}}
$$

Here following (2.51), we write

$$
\left(P^{\delta, \widetilde{\varepsilon}}-\widetilde{P}^{\delta}\right)\left(1-\chi_{\sqrt{\tilde{\varepsilon}}}\right)\left(z-\widetilde{P}^{\delta}\right)^{-1} \chi_{\sqrt{\widetilde{\varepsilon}}}
$$

as

$$
\left(P^{\delta, \widetilde{\varepsilon}}-\widetilde{P}^{\delta}\right)\left(1-\chi_{\sqrt{\widetilde{\varepsilon}}}\right) \Lambda^{-k-2}\left(\Lambda^{k+2}\left(z-\widetilde{P}^{\delta}\right)^{-1} \Lambda^{-k}\right) \Lambda^{k} \chi_{\sqrt{\widetilde{\varepsilon}}} .
$$

Here using (2.24) we see that

$$
\left(P^{\delta, \widetilde{\varepsilon}}-\widetilde{P}^{\delta}\right)\left(1-\chi_{\sqrt{\widetilde{\varepsilon}}}\right) \Lambda^{-k-2}=\mathcal{O}\left(\frac{h^{k / 2+1}}{\widetilde{\varepsilon}^{k / 2}}\right) .
$$

On the other hand, as in (2.50),

$$
\Lambda^{k+2}\left(z-\widetilde{P}^{\delta}\right)^{-1} \Lambda^{-k}=\mathcal{O}\left(\frac{1}{h}\right): L^{2} \rightarrow L^{2},
$$


and also,

$$
\Lambda^{k} \chi_{\sqrt{\widetilde{\varepsilon}}}=\mathcal{O}\left(\frac{\widetilde{\varepsilon}^{k / 2}}{h^{k / 2}}\right): L^{2} \rightarrow L^{2} .
$$

Combining (2.60), (2.61), (2.62), and (2.63), we see that the expression (2.59) is $\mathcal{O}(1)$.

We now come to estimate the remaining factor in (2.58). To that end, we let

$$
L_{\widetilde{\varepsilon}}=\widetilde{\mathcal{O}}\left(1+\frac{\min \left((x, \xi)^{2}, \widetilde{\varepsilon}\right)}{h}\right)
$$

be an elliptic symbol in the class defined by the right hand side, and write

$$
\left(z-P^{\delta, \widetilde{\varepsilon}}\right)^{-1}\left(1-\chi_{\sqrt{\widetilde{\varepsilon}}}\right)=\left(\left(z-P^{\delta, \widetilde{\varepsilon}}\right)^{-1} L_{\widetilde{\varepsilon}}\right) L_{\widetilde{\varepsilon}}^{-1}\left(1-\chi_{\sqrt{\widetilde{\varepsilon}}}\right) .
$$

Here we know that

$$
\left(z-P^{\delta, \widetilde{\varepsilon}}\right)^{-1} L_{\widetilde{\varepsilon}}=\mathcal{O}\left(\frac{1}{h}\right): L^{2} \rightarrow L^{2},
$$

and since

$$
L_{\widetilde{\varepsilon}}^{-1}\left(1-\chi_{\sqrt{\widetilde{\varepsilon}}}\right)=\mathcal{O}\left(\frac{h}{\widetilde{\varepsilon}}\right): L^{2} \rightarrow L^{2},
$$

it follows that the expression (2.64) is $\mathcal{O}\left(\frac{1}{\tilde{\varepsilon}}\right)$. Using finally that the length of the integration contour in (2.55) is $\mathcal{O}_{B}(h)$, we get

$$
\mathrm{II}=\mathcal{O}_{B}\left(\frac{h}{\widetilde{\varepsilon}}\right)=\mathcal{O}_{B}\left(\frac{1}{\widetilde{A}}\right): L^{2} \rightarrow L^{2} .
$$

Combining (2.55) $,(2.56),(2.65)$, we conclude that

$$
\left(\Pi_{B}-\widetilde{\Pi}_{B}\right) \chi_{\sqrt{\widetilde{\varepsilon}}}=\mathcal{O}_{B}\left(\frac{1}{\widetilde{A}}\right): L^{2} \rightarrow L^{2} .
$$

In view of (2.54), the bound (2.47) follows, and this completes the proof of Lemma 2.1.

We now come to estimate the second term in the right hand side of (2.31), given by $K_{\varepsilon}\left(1-\Pi_{0, B}\right) u$. Here, the difficulty is that in general, due to a pseudospectral phenomenon [3], the operator norm of $\Pi_{0, B}$ may exhibit some exponential growth, as 
$h \rightarrow 0$. To circumvent this issue, our fist task will be to establish a more manageable characterization of the vector $v=\left(1-\Pi_{0, B}\right) u$. Specifically, we shall now discuss properties of the range of the projection $1-\Pi_{0, B}$ on $L^{2}$.

In (2.27), following [20], we have already recalled the form of the eigenvalues of the elliptic quadratic operator $P_{0}$. From [20], we know furthermore that the generalized eigenfunctions of $P_{0}$ are of the form

$$
h^{-n / 4} p\left(\frac{x}{\sqrt{h}}\right) e^{i \Phi(x) / h}
$$

where $p(x)$ is a polynomial on $\mathbb{R}^{n}$ and $\Phi(x)$ is a quadratic form with $\operatorname{Im} \Phi>0$. The degree of the polynomial $p(x)$ in (2.66) tends to $\infty$ together with the real part of the eigenvalue $h \mu_{j, k}$ in (2.27). We may also recall from [20] that $\Phi$ in (2.66) is such that the positive Lagrangian subspace $\Lambda_{\Phi}=\left\{\left(x, \Phi^{\prime}(x)\right), x \in \mathbb{C}^{n}\right\}$ is the direct sum of the generalized eigenspaces of the Hamilton map of $p_{0}$, corresponding to the eigenvalues with a positive imaginary part. Correspondingly, the generalized eigenfunctions of the formal $L^{2}$ adjoint $P_{0}^{*}$ are of the form

$$
h^{-n / 4} q\left(\frac{x}{\sqrt{h}}\right) e^{i \Psi(x) / h}
$$

where $q$ is a polynomial and $\Psi$ is a quadratic form such that $\operatorname{Im} \Psi$ is positive definite.

Let $e_{1}, \ldots e_{N}$ be a basis for $\operatorname{Ran}\left(\Pi_{0, B}\right)$ and let $e_{1}^{*}, \ldots e_{N}^{*}$ be the corresponding dual basis for $\operatorname{Ran}\left(\left(\Pi_{0, B}\right)^{*}\right)$. If $v \in L^{2}$, we have

$$
\Pi_{0, B} v=\sum_{j=1}^{N}\left(v \mid e_{j}^{*}\right) e_{j}
$$

and therefore, $v \in \operatorname{Ran}\left(1-\Pi_{0, B}\right)$ precisely when $v$ is orthogonal to $\operatorname{Ran}\left(\left(\Pi_{0, B}\right)^{*}\right)$.

Proposition 2.2 There exists a selfadjoint $h$-differential operator $Q=\mathrm{Op}_{h}(q)$, where $q$ is a positive definite quadratic form on $T^{*} \mathbb{R}^{n}$, such that

$$
\operatorname{Ran}\left(E\left(Q, \frac{B h}{C}\right)\right) \subset \operatorname{Ran}\left(\left(\Pi_{0, B}\right)^{*}\right) \subset \operatorname{Ran}(E(Q, C B h)),
$$

for some $C>1$ which is independent of $Q$ and $B$. Here $E(Q, \lambda)=1_{(-\infty, \lambda]}(Q)$ is the finite rank spectral projection associated to $Q$ and the interval $(-\infty, \lambda]$. 
Proof: The operator $Q$ will be seen to be essentially the $h$-Weyl quantization of the classical harmonic oscillator on $\mathbb{R}^{n}$. When constructing $Q$, recall that the generalized eigenfunctions of $P_{0}^{*}$ are of the form (2.67). We shall write $\Psi(x)=(B x, x)$, where $B$ is a symmetric matrix, $B=B_{1}+i B_{2}$, where $B_{j}$ are real, $j=1,2$, and $B_{2}>0$. The real linear canonical transformation

$$
\kappa_{1}:(x, \xi) \mapsto\left(x, \xi-B_{1} x\right)
$$

maps the positive Lagrangian subspace $\Lambda_{\Psi}=\left\{(x, B x) ; x \in \mathbb{C}^{n}\right\}$ to the positive Lagrangian subspace $\left\{\left(x, i B_{2} x\right) ; x \in \mathbb{C}^{n}\right\}$. Now since $B_{2}>0$, there exists an invertible real $n \times n$ matrix $C$ such that the real linear canonical transformation

$$
\kappa_{2}:(x, \xi) \mapsto\left(C^{-1} x, C^{t} \xi\right)
$$

maps $\left\{\left(x, i B_{2} x\right) ; x \in \mathbb{C}^{n}\right\}=\kappa_{1}\left(\Lambda_{\Psi}\right)$ to $\left\{(x, i x) ; x \in \mathbb{C}^{n}\right\}$. We take the operator

$$
\widetilde{Q}=\frac{1}{2} \sum_{j=1}^{n}\left(x_{j}^{2}+\left(h D_{x_{j}}\right)^{2}\right)=\mathrm{Op}_{h}(\widetilde{q}), \quad \widetilde{q}(x, \xi)=\frac{1}{2} \sum_{j=1}^{n}\left(x_{j}^{2}+\xi_{j}^{2}\right),
$$

associated to $\Lambda_{\widetilde{\varphi}}=\left\{(x, i x) ; x \in \mathbb{C}^{n}\right\}=\left(\kappa_{2} \circ \kappa_{1}\right)\left(\Lambda_{\Psi}\right), \widetilde{\varphi}(x)=i x^{2} / 2$. To obtain the operator $Q$ it only remains to notice that associated to $\kappa_{1}$ and $\kappa_{2}$ we have the metaplectic operators

$$
U_{1}: L^{2} \rightarrow L^{2}, \quad U_{1} f(x)=e^{-i\left(B_{1} x, x\right) / 2 h} f(x),
$$

and

$$
U_{2}: L^{2} \rightarrow L^{2}, \quad U_{2} f(x)=f(C x)|\operatorname{det} C|^{1 / 2},
$$

both unitary on $L^{2}$, and hence with $U:=U_{2} \circ U_{1}$, we can take $Q:=U^{-1} \widetilde{Q} U=$ $\mathrm{Op}_{h}\left(\left(\widetilde{q} \circ\left(\kappa_{2} \circ \kappa_{1}\right)\right)\right)$. Notice that the eigenfunctions of $Q$ are of the form

$$
e_{\alpha, h}(x)=e_{\alpha, h=1}\left(\frac{x}{\sqrt{h}}\right), \quad e_{\alpha, h=1}(x)=H_{\alpha}\left(C^{-1} x\right) e^{i \Psi(x)}, \quad Q e_{\alpha, h}=h\left(|\alpha|+\frac{n}{2}\right) e_{\alpha, h}
$$

where $H_{\alpha}(x)=\prod_{j=1}^{n} H_{\alpha_{j}}\left(x_{j}\right), \alpha \in \mathbb{N}^{n}$, are the Hermite polynomials. The result follows.

Having established a favorable comparison for the linear space $\operatorname{Ran}\left(\left(\Pi_{0, B}\right)^{*}\right) \subset$ $L^{2}$, we return to the problem of estimating $K_{\varepsilon} v$, for $v=\left(1-\Pi_{0, B}\right) u$. Let $\psi \in$ $C^{\infty}(\mathbb{R} ;[0,1])$ with $\operatorname{supp}(\psi) \subset[0,1]$, and set

$$
\psi_{\lambda}(t)=\psi\left(\frac{t}{\lambda}\right), \quad \lambda>0
$$


It follows then from Proposition 2.2 that

$$
\psi_{\frac{B h}{C}}(Q) v=0 .
$$

To understand the operator occurring in (2.77), it is convenient to perform a suitable dilation in phase space. Assume therefore that $\lambda>0$ in (2.76) is such that $h \ll \lambda \ll$ 1. Let us make the change of variables

$$
x=\lambda^{1 / 2} \widetilde{x}, \quad D_{x}=\lambda^{-1 / 2} D_{\widetilde{x}}
$$

Then, since the operator $Q$ is quadratic,

$$
\frac{1}{\lambda} Q=\frac{1}{\lambda} q^{w}\left(x, h D_{x}\right)=\frac{1}{\lambda} q^{w}\left(\lambda^{1 / 2}\left(\widetilde{x}, \frac{h}{\lambda} D_{\widetilde{x}}\right)\right)=q^{w}\left(\widetilde{x}, \frac{h}{\lambda} D_{\widetilde{x}}\right) .
$$

It follows therefore from the functional calculus in the version of [4] that

$$
\psi\left(\lambda^{-1} Q\right)=\mathrm{Op}_{\frac{h}{\lambda}, \widetilde{x}}\left(r\left(\widetilde{x}, \widetilde{\xi} ; \frac{h}{\lambda}\right)\right)=r^{w}\left(\lambda^{-1 / 2}\left(x, h D_{x}\right) ; \frac{h}{\lambda}\right)
$$

where $r \in S\left(\langle\cdot\rangle^{-N}\right)$ for any $N \in \mathbb{N}$, with a complete asymptotic expansion in each of these symbol spaces, and with the leading symbol $\psi(q(\widetilde{x}, \widetilde{\xi}))$.

Remark. It is well known [14] that when $Q=\mathrm{Op}_{h}^{w}(q)$ where $q$ is a positive definite quadratic form, then the Weyl symbol of $f(Q), f \in C_{0}^{\infty}(\mathbb{R})$, is of the form $\tilde{f}(q ; h)=$ $f(q)+\mathcal{O}(h)$. It follows therefore that in (2.79) we have

$$
r\left(\widetilde{x}, \widetilde{\xi} ; \frac{h}{\lambda}\right)=\widetilde{\psi}\left(q(\widetilde{x}, \widetilde{\xi}) ; \frac{h}{\lambda}\right)
$$

where the leading term of $\widetilde{\chi}$ is $\psi(q(\widetilde{x}, \widetilde{\xi}))$.

It is therefore clear that in (2.25) we can take

$$
K_{\varepsilon}=\varepsilon \psi_{\frac{B h}{C}}(Q), \quad \varepsilon=A h,
$$

for a suitable choice of $B \geq A$ fixed, where we can take $B$ as a fixed multiple of $A$. With this choice, we get, using (2.77),

$$
K_{\varepsilon}\left(1-\Pi_{0, B}\right) u=0 .
$$


Combining (2.26), (2.31), Lemma 2.1, and (2.81), we see that for $u \in L^{2}$ with $u \in \operatorname{Ran}\left(1-\Pi_{B}\right)$, we have

$$
\left(\frac{\delta \varepsilon}{C}-C h\right)\|u\|^{2} \leq \operatorname{Re}\left(P^{\delta, \widetilde{\varepsilon}} u, u\right)+\varepsilon \mathcal{O}_{B}\left(\widetilde{A}^{3 / 2} h^{1 / 2}+\widetilde{A}^{-1}\right)\|u\|^{2} .
$$

Recall that here $B \geq A, B=\mathcal{O}(A)$, is taken fixed, and $\delta>0$ is sufficiently small but fixed. Choosing first $\widetilde{A} \gg B$ large enough and then taking $h$ sufficiently small depending on these parameters, we absorb the second term in the right hand side of (2.82) into the left hand side.

Proposition 2.3 When $A \leq B \ll \widetilde{A}$, let $\Pi_{B}$ be the spectral projection of $P^{\delta, \widetilde{\varepsilon}}$, $\widetilde{\varepsilon}=\widetilde{A} h$, associated with $D(0, B h)$. Here $B$ is a fixed multiple of $A$. Assume that $u \in \mathcal{D}\left(P^{\delta, \widetilde{\varepsilon}}\right)$ is such that $u \in \operatorname{Ran}\left(1-\Pi_{B}\right)$. Then for $h$ sufficiently small, we have

$$
\operatorname{Re}\left(P^{\delta, \widetilde{\varepsilon}} u \mid u\right) \geq \frac{B h}{\mathcal{O}(1)}\|u\|^{2}, \quad \widetilde{\varepsilon}=\widetilde{A} h .
$$

Now recall that

$$
P^{\delta, \widetilde{\varepsilon}}=A_{\delta, \widetilde{\varepsilon}}^{-1} P A_{\delta, \widetilde{\varepsilon}}
$$

where $A_{\delta, \widetilde{\varepsilon}}, A_{\delta, \widetilde{\varepsilon}}^{-1}: \mathcal{S} \rightarrow \mathcal{S}, L^{2} \rightarrow L^{2}$, have $L^{2}$ norm $\mathcal{O}_{\widetilde{A}}(1)$. It is therefore clear from Proposition 2.3 that if $u$ is such that $u \in \operatorname{Ran}(1-\Pi)$, where

$$
\Pi=\frac{1}{2 \pi i} \int_{h \gamma}(z-P)^{-1} d z
$$

is the spectral projection of $P$ associated with the spectrum of $P$ in $D(0, B h)$, then

$$
\left\|e^{-t P / h} u\right\| \leq \mathcal{O}(1) e^{-t / C}\|u\|, \quad C=C(B)>0 .
$$

Therefore, it only remains to consider the restriction of the semigroup $e^{-t P / h}$ to the finite-dimensional subspace $\operatorname{Ran}(\Pi)$, generated by the generalized eigenfunctions of $P$ corresponding to the eigenvalues of $P$ of modulus $<B h$. We shall now proceed to do so, in the framework of supersymmetric differential operators.

\section{Supersymmetric operators and return to equi- librium in the double well case}

The purpose of this section is to establish Theorem 1.1 in its general form, for a class of supersymmetric second order differential operators, including (1.1). Specifically, let

$$
A: \mathbb{R}^{n} \rightarrow \mathbb{R}^{n}
$$


be an invertible constant matrix. We decompose

$$
A=B+C, \quad{ }^{t} B=B, \quad{ }^{t} C=-C,
$$

and assume that

$$
B \geq 0
$$

When $\phi \in C^{\infty}\left(\mathbb{R}^{n} ; \mathbb{R}\right)$ is a Morse function such that

$$
\partial_{x}^{\alpha} \phi(x)=\mathcal{O}(1), \quad \partial_{x}^{\alpha}\left(\left\langle B \partial_{x} \phi, \partial_{x} \phi\right\rangle\right)=\mathcal{O}(1), \quad|\alpha| \geq 2,
$$

we consider the Witten-Hodge Laplacian associated to $A$ and $\phi$, acting on scalar functions, defined as in section 10 of [12],

$$
\begin{aligned}
P=-\Delta_{A}^{(0)}= & \quad \sum_{j, k} h D_{x_{j}} B_{j, k} h D_{x_{k}}+\sum_{j, k}\left(\partial_{x_{j}} \phi\right) B_{j, k}\left(\partial_{x_{k}} \phi\right)-h \operatorname{tr}\left(B \phi^{\prime \prime}\right) \\
& +\sum_{j, k}\left(\left(\partial_{x_{k}} \phi\right) C_{j, k} h \partial_{x_{j}}+h \partial_{x_{j}} \circ C_{j, k}\left(\partial_{x_{k}} \phi\right)\right) .
\end{aligned}
$$

The principal symbol of $P$ is of the form

$$
p(x, \xi)=\langle B \xi, \xi\rangle+2 i\left\langle C \phi_{x}^{\prime}, \xi\right\rangle+\left\langle B \phi_{x}^{\prime}, \phi_{x}^{\prime}\right\rangle,
$$

so that the assumptions (2.5), (2.6), (2.7) are satisfied.

Assume that the Morse function $\phi$ has finitely many critical points $x_{1}, \ldots x_{N} \in \mathbb{R}^{n}$ and that

$$
\left|\phi^{\prime}(x)\right| \geq \frac{1}{C}, \quad|x| \geq C .
$$

The assumption (2.10) holds with

$$
\mathcal{C}=\left\{\rho_{j} ; j=1, \ldots N\right\}, \quad \rho_{j}=\left(x_{j}, 0\right),
$$

and we shall also assume that the dynamical assumptions (2.14), (2.15), and (2.16) are valid. We then know that the results of section 2 can be applied to $P$ in (3.5) .

As in [12, we shall assume now that

$$
\phi \text { has precisely three critical points, of which }
$$

two are local minima $U_{ \pm 1}$, and the third one $U_{0}$ is of index one.

Then we know from 12 that for $C>0$ large enough, $P$ in (3.5) has precisely 2 eigenvalues $\mu_{0}=0$ and $\mu_{1}$ in the $\operatorname{disc} D(0, h / C)$ for $h$ small enough. Here $\mu_{1}$ is real and such that

$$
\mu_{1}=h\left(a_{1}(h) e^{-2 S_{1} / h}+a_{-1}(h) e^{-2 S_{-1} / h}\right), \quad S_{j}=\phi\left(U_{0}\right)-\phi\left(U_{j}\right)>0, \quad j= \pm 1,
$$


where $a_{j}(h)$ are real, $a_{j}(h) \sim a_{j, 0}+h a_{j, 1}+\ldots, a_{j, 0}>0$.

The set $\phi^{-1}\left(\left(-\infty, \phi\left(U_{0}\right)\right)\right)$ has precisely two connected components $D_{j}, j= \pm 1$, determined by the condition $U_{j} \in D_{j}$. Let $0 \leq \chi_{j} \in C_{0}^{\infty}\left(D_{j}\right)$ be such that $\chi_{j}=1$ on $D_{j} \cap \phi^{-1}\left(\left(-\infty, \phi\left(U_{0}\right)-\varepsilon_{0}\right)\right)$ for $\varepsilon_{0}>0$ fixed but arbitrarily small. If

$$
f_{j}=h^{-n / 4} c_{j}(h) e^{-\frac{1}{h}\left(\phi(x)-\phi\left(U_{j}\right)\right)} \chi_{j}(x), \quad j= \pm 1,
$$

where $c_{j}(h)>0$ is a normalization constant such that $\left\|f_{j}\right\|=1$, and

$$
\Pi=\frac{1}{2 \pi i} \int_{\gamma}(z-P)^{-1} d z, \quad \gamma=\partial D\left(0, \frac{h}{C}\right), \quad C>0,
$$

is the rank 2 spectral projection of $P$ corresponding to the eigenvalues $\mu_{0}=0$ and $\mu_{1}$ in (3.9), we have the basis

$$
e_{j}=\Pi f_{j}, \quad j= \pm 1,
$$

for $\operatorname{Ran}(\Pi)$, introduced in [12]. From section 11 of 12 we recall that

$$
e_{j}=f_{j}+\mathcal{O}\left(h^{-N_{1}} e^{-\frac{1}{h}\left(S_{j}-\varepsilon_{0}\right)}\right) \quad \text { in } L^{2}, \quad N_{1}>0,
$$

and that the restriction of $P$ to the space $\operatorname{Ran}(\Pi)$ has the matrix

$$
\left(\begin{array}{c}
\lambda_{-1}^{*} \\
\lambda_{1}^{*}
\end{array}\right)\left(\begin{array}{ll}
\lambda_{-1} & \lambda_{1}
\end{array}\right)=\left(\begin{array}{cc}
\lambda_{-1}^{*} \lambda_{-1} & \lambda_{-1}^{*} \lambda_{1} \\
\lambda_{1}^{*} \lambda_{-1} & \lambda_{1}^{*} \lambda_{1}
\end{array}\right),
$$

with respect to the basis $\left(e_{-1}, e_{1}\right)$, with the eigenvalues $\mu_{0}=0$ and

$$
\mu_{1}=\lambda_{-1}^{*} \lambda_{-1}+\lambda_{1}^{*} \lambda_{1} .
$$

A simple computation shows that a corresponding basis of the eigenvectors is given by

$$
\lambda_{1} e_{-1}-\lambda_{-1} e_{1}
$$

and

$$
\lambda_{-1}^{*} e_{-1}+\lambda_{1}^{*} e_{1} .
$$

Here we recall from the formulas (11.43), (11.45), and the following discussion in [12] that if $\left|\lambda_{1}\right| \geq \frac{1}{C}\left|\lambda_{-1}\right|$ then $\left|\lambda_{1}^{*}\right| \geq \frac{1}{2 C}\left|\lambda_{-1}^{*}\right|$ and $\lambda_{1} \lambda_{1}^{*}>0$. We have the same fact after permuting the indices $-1,1$ and the $\lambda_{j}, \lambda_{j}^{*}$. It follows that

$$
\mu_{1} \sim \max \left|\lambda_{j}\right|^{2} \sim \max \left|\lambda_{j}^{*}\right|^{2} .
$$


Rather than using (3.11) and (3.12), we shall make a normalized choice of the eigenfunctions, given by

$$
v_{0}=\frac{1}{\sqrt{\mu_{1}}}\left(\lambda_{1} e_{-1}-\lambda_{-1} e_{1}\right)
$$

and

$$
v_{1}=\frac{1}{\sqrt{\mu_{1}}}\left(\lambda_{-1}^{*} e_{-1}+\lambda_{1}^{*} e_{1}\right) .
$$

The corresponding matrix of the coefficients is given by

$$
V=\frac{1}{\sqrt{\mu_{1}}}\left(\begin{array}{cc}
\lambda_{1} & -\lambda_{-1} \\
\lambda_{-1}^{*} & \lambda_{1}^{*}
\end{array}\right) .
$$

We have $\operatorname{det} V=1$ and it follows from 3.13 that $V=\mathcal{O}(1)$. Hence the inverse matrix $V^{-1}$ has the same properties, so that $v_{0}, v_{1}$ is a well-behaved basis of eigenfunctions for $P$. If $\left(e_{-1}^{*}, e_{1}^{*}\right) \in \operatorname{Ran}\left(\Pi^{*}\right)$ is the basis that is dual to $\left(e_{-1}, e_{1}\right)$, then the corresponding basis of eigenfunctions of $P^{*}$, dual to $\left(v_{0}, v_{1}\right)$ is given by the matrix ${ }^{t} V^{-1}$, so that

$$
v_{0}^{*}=\frac{1}{\sqrt{\mu_{1}}}\left(\lambda_{1}^{*} e_{-1}^{*}-\lambda_{-1}^{*} e_{1}^{*}\right)
$$

and

$$
v_{1}^{*}=\frac{1}{\sqrt{\mu_{1}}}\left(\lambda_{-1} e_{-1}^{*}+\lambda_{1} e_{1}^{*}\right) .
$$

We summarize the discussion above in the following proposition.

Proposition 3.1 Let $v_{j}$ and $v_{j}^{*}$ be defined as in (3.14),(3.15), (3.17), (3.18). Then the spectral projections

$$
\Pi_{j}=\left(\cdot \mid v_{j}^{*}\right) v_{j}, \quad j=0,1
$$

associated to the eigenvalues $\mu_{0}=0$ and $\mu_{1}$ in (3.9) are uniformly bounded as $h \rightarrow 0$.

Combining (2.85) together with Proposition 3.1, as well as with Theorem 8.4 of [12], we get the result in Theorem 1.1 in the general case.

Theorem 3.2 Let $P=-\Delta_{A}^{(0)}$ where we assume (3.1)3.4), (3.7), and (3.8). We also assume that $P$ satisfies the dynamical hypotheses (2.10), (2.14), (2.15), so that the disc $D(0, h / C)$ for $C>0$ large enough, contains precisely 2 eigenvalues of $P, \mu_{0}=0$ 
and $\mu_{1}$ given in (3.9). Let $\Pi_{j}$ be the spectral projection associated with the eigenvalue $\mu_{j}, j=0,1$. Then we have

$$
\Pi_{j}=\mathcal{O}(1), \quad h \rightarrow 0 .
$$

We have furthermore, uniformly as $t \geq 0$ and $h \rightarrow 0$,

$$
e^{-t P / h}=\Pi_{0}+e^{-t \mu_{1} / h} \Pi_{1}+\mathcal{O}(1) e^{-t / C}, \quad C>0, \quad \text { in } \quad \mathcal{L}\left(L^{2}, L^{2}\right) .
$$

Here we have also used that the eigenvalues of $P$ in $D(0, B h) \backslash D(0, h / C)$ have real parts $\geq h / \mathcal{O}(1)$.

\section{Tunnel effect for a well and the sea}

In this section we shall show how to adapt the analysis of section 11 of [12] and that of section 3 of the present work to cover the case of a potential with a single well and a saddle point, rather than a double well and a saddle point as before. Some parts of this section are very close to the corresponding ones of section 8 in [12], and rather than repeating the arguments, we shall often merely refer to the discussion there.

As in section 3, we shall consider the supersymmetric case. Assume that we are given the constant matrices $A=B+C$ and a Morse function $\phi$, that satisfy (3.1) - (3.4). We then have the corresponding Witten-Hodge Laplacian in degree 0, given by (3.5), with a principal symbol (3.6) so that the assumptions (2.5)-(2.7) hold. We refer to the formula (11.3) of [12] for the more general expression for the Witten-Hodge Laplacian in degree $q \geq 0, P^{(q)}$.

As before, we shall assume that $\phi$ has finitely many critical points $x_{1}, \ldots, x_{N} \in \mathbb{R}^{n}$ and that $\left|\phi^{\prime}(x)\right| \geq 1 / C$ when $|x| \geq C$, with $C$ large. The assumptions (2.10) is therefore satisfied with $\mathcal{C}=\left\{\rho_{j} ; j=1, \ldots, N\right\}$ where $\rho_{j}=\left(x_{j}, 0\right)$. We also assume that the dynamical assumptions (2.14), (2.15), (2.16) hold.

As in section 11 of [12], an application of Theorem 8.3 of [12] to $P^{(q)}$ shows that the eigenvalues $\mu_{j, k}$ there are of the form

$$
\mu_{j, k}=\frac{1}{i} \sum_{k=1}^{n}\left(\nu_{j, k, \ell}+\frac{1}{2}\right) \lambda_{\ell}+\gamma_{j, k}
$$

where $\gamma_{j, k}$ is any eigenvalue of the subprincipal symbol $S_{P^{(q)}}$ at $\left(x_{j}, 0\right)$. From the calculations in subsection 10.3 of [12] we recall that the $\mu_{j, k}$ will be confined to a sector $\{0\} \cup\{|\arg z|<\pi / 2-1 / C\}$ around $[0,+\infty)$. Recall that it is precisely when $x_{j}$ is of index $q$ (i.e. when the Hessian of $\phi$ at $x_{j}$ has precisely $q$ negative eigenvalues) that one of the $\mu_{j, k}$ is equal to 0 . 
We shall now introduce more specific conditions for the case that we study here. Instead of assuming that we are in the double well case, let us shall suppose that we have a single well and a sea, that is

$\phi$ has precisely two critical points, one local minimum $U_{1}$, and a "saddle point" $U_{0}$ of index one.

Notice that this implies that the Maxwellian $e^{-\phi / h}$ is no longer an eigenfunction of $P^{(0)}$, since $\phi(x)$ does not go to $+\infty$ with $|x|$.

Put $S_{1}=\phi\left(U_{0}\right)-\phi\left(U_{1}\right)$ so that $S_{1}>0$. The set $\phi^{-1}(]-\infty, \phi\left(U_{0}\right)[)$ has precisely two connected components $D_{j}, j= \pm 1$, where $D_{1}$ is determined by the condition that $U_{1} \in D_{1}$, while $D_{-1}$ is unbounded.

Under these assumptions we shall prove the following result:

Theorem 4.1 Let $P=-\Delta_{A}^{(0)}$ be as in (3.5), where we assume (4.2). Then for $C>0$ large enough, $P$ has precisely 1 eigenvalue $\mu_{1}$ in the disc $D(0, h / C)$ when $h>0$ is small enough. Here $\mu_{1}$ is real and of the form

$$
\mu_{1}=h a_{1}(h) e^{-2 S_{1} / h}
$$

where $a_{1}(h)$ are real, $a_{1}(h) \sim a_{1,0}+a_{1,1} h+\ldots, a_{1,0}>0, S_{1}=\phi\left(U_{0}\right)-\phi\left(U_{1}\right)$.

Remark 4.2. It is clear that Theorem 4.1 implies an analog of Theorem 3.2 in the present metastable case. We shall refrain from formulating it explicitly.

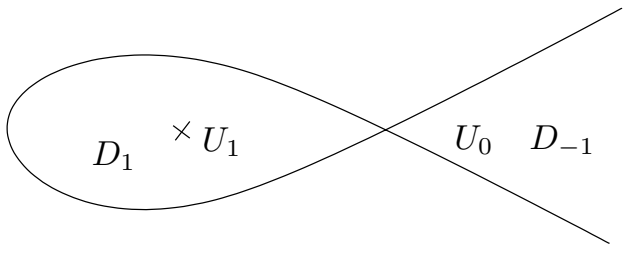

level line at $\phi\left(U_{0}\right)$

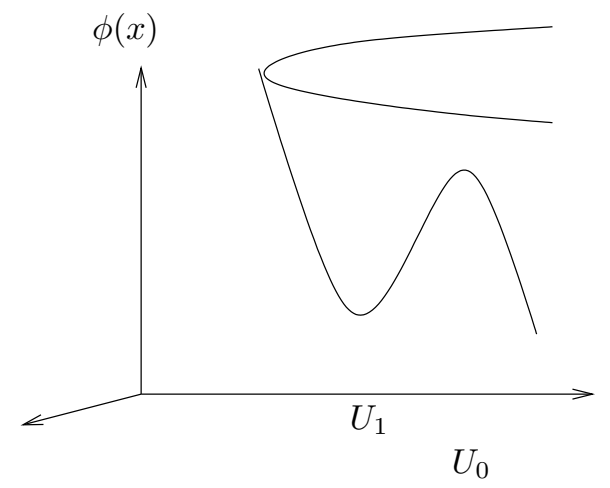

Figure 1: A well and the sea

Proof: We first know that $P^{(0)}=-\Delta_{A}^{(0)}$ has precisely one eigenvalue $\mu_{1}=o(h)$ spanning a corresponding 1-dimensional spectral subspace $E^{(0)}$ since there is a unique 
local minimum for $\phi$. Now while $e^{-\phi / h}$ does not belong to $L^{2}$, a truncation of this function can be used as a quasimode near $U_{1}$ and it follows therefore as in [12], that $\mu_{1}=\mathcal{O}\left(h^{\infty}\right)$. Moreover, $-\Delta_{A}^{(1)}$ has precisely one eigenvalue $\widetilde{\mu}_{1}=o(h)$ and $-\Delta_{A}^{(k)}$ has no eigenvalue $=o(h)$ for $k \geq 2$ from the discussion in the beginning of the paragraph. Since our operators are real we know that the spectra are symmetric around the real axis, hence $\mu_{1}, \widetilde{\mu}_{1}$ are real. From the intertwining relations

$$
-\Delta_{A}^{(1)} d_{\phi}=d_{\phi}\left(-\Delta_{A}^{(0)}\right),-\Delta_{A}^{(0)} d_{\phi}^{A, *}=-d_{\phi}^{A, *} \Delta_{A}^{(1)},
$$

we then also know that $\widetilde{\mu}_{1}=\mu_{1}$ (see also the discussion at the end of page 69 of [12]).

The construction of the eigenfunctions $e_{0}$ and $e_{1}$ associated to the critical points $U_{1}$ and $U_{0}$ for respectively $-\Delta_{A}^{(1)}$ and $-\Delta_{A}^{(0)}$ is exactly the same as in [12]. We only retain the following from there:

We begin with $-\Delta_{A}^{(0)}$. Let $\chi_{1} \in C_{0}^{\infty}\left(D_{1}\right)$ be equal to 1 on $D_{1} \cap \phi^{-1}(]-\infty, \phi\left(U_{0}\right)-$ $\left.\left.\epsilon_{0}\right]\right)$ for $\epsilon_{0}>0$ fixed but arbitrarily small. Consider

$$
f_{1}=h^{-n / 4} c_{1}(h) e^{-\frac{1}{h}\left(\phi(x)-\phi\left(U_{1}\right)\right)} \chi_{1}(x),
$$

where $c_{1}(h) \sim c_{1,0}+h c_{1,1}+\ldots>0$ is a normalization constant with $c_{1,0}>0$, such that $\left\|f_{1}\right\|=1$. Then the normalized eigenfunction associated to $\mu_{1}$ is given by

$$
e_{1}:=f_{1}+\mathcal{O}\left(h^{-N_{1}} e^{-\frac{1}{h}\left(S_{1}-\epsilon_{0}\right)}\right) \text { in } L^{2} .
$$

We continue with the study of $-\Delta_{A}^{(0)}$. Let $E^{(1)}$ be the one-dimensional eigenspace of $P^{(1)}$ corresponding to $\mu_{1}$. From an easy extension of [12, Theorem 9.1] (see also Remark 9.2 there) to the non-scalar case with the presence of the other non-resonant well $U_{1}$, we know that $E^{(1)}$ is generated by an eigenform

$$
e_{0}(x ; h)=\chi_{0}(x) e^{-\frac{1}{h} \phi_{+}(x)} h^{-\frac{n}{4}} a_{0}(x ; h)+\mathcal{O}\left(e^{-S_{0} / h}\right),
$$

where $\chi_{0} \in C_{0}^{\infty}\left(\operatorname{neigh}\left(U_{0}\right)\right)$ is equal to one near $U_{0}, S_{0}>0$, and

$$
a_{0}(x ; h) \sim \sum_{0}^{\infty} a_{0, k}(x) h^{k}
$$

is a symbol as in Theorem 9.1 of [12], with $a_{0,0}\left(U_{0}\right) \neq 0$. Here the phase $\phi_{+} \in$ $C^{\infty}\left(\right.$ neigh $\left.\left(U_{0}\right) ;[0, \infty)\right)$ satisfies $\phi_{+}(x) \backsim\left|x-U_{0}\right|^{2}$ and solves the eikonal equation $q\left(x, \phi_{+}^{\prime}(x)\right)=0$, with $q=p_{2}+p_{1}-p_{0}$.

From [12], let us recall that the phase function $\phi_{+}$arises as the generating function for the stable outgoing manifold through $\left(U_{0}, 0\right)$ for the $H_{q^{-}}$flow, $\Lambda_{\Phi_{+}}$, and recall also 
that $\phi_{+}^{\prime \prime}\left(U_{0}\right)>0$ by Proposition [12, Proposition 8.2]. (Similarly we have a stable incoming manifold $\Lambda_{\phi_{-} .}$) Let $k_{ \pm}$be the number of eigenvalues of the linearization of $H_{q_{\Lambda_{\phi}}}$ at that point with \pm real part $>0$, so that $k_{+}+k_{-}=n$. Let $K_{+}, K_{-} \subset \Lambda_{\phi}$ be the corresponding stable outgoing and incoming submanifolds of dimension $k_{+}$ and $k_{-}$respectively. Then $K_{+} \subset \Lambda_{\phi_{+}}, K_{-} \subset \Lambda_{\phi_{-}}$and $\phi-\phi\left(U_{0}\right)-\phi_{ \pm}$vanishes to the second order on $\pi_{x}\left(K_{ \pm}\right)$. Since $\phi^{\prime \prime}\left(U_{0}\right)$ has signature $(n-1,1)$, we conclude that $\operatorname{dim} K_{+}=n-1, \operatorname{dim} K_{-}=1$. It is also clear that $\Lambda_{\phi}, \Lambda_{\phi_{ \pm}}$intersect cleanly along $K_{ \pm}$, so we get

$$
\begin{aligned}
\phi_{+}-\left(\phi-\phi\left(U_{0}\right)\right) & \sim \operatorname{dist}\left(x, \pi_{x}\left(K_{+}\right)\right)^{2}, \\
\phi-\phi\left(U_{0}\right)-\phi_{-} & \backsim \operatorname{dist}\left(x, \pi_{x}\left(K_{-}\right)\right)^{2} .
\end{aligned}
$$

We now make some remarks about the adjoint operator $-\Delta_{t_{A}}=\left(-\Delta_{A}\right)^{t} A, *$. As proved in [12, Subsection 10.4]), we get the corresponding phases and submanifolds in this case, which satisfy

$$
\begin{aligned}
& \phi_{+}^{*}-\left(\phi-\phi\left(U_{0}\right)\right) \backsim \operatorname{dist}\left(x, \pi_{x}\left(K_{+}^{*}\right)\right)^{2}, \\
& \phi-\phi\left(U_{0}\right)-\phi_{-}^{*} \backsim \operatorname{dist}\left(x, \pi_{x}\left(K_{-}^{*}\right)\right)^{2} \text {. }
\end{aligned}
$$

and for symmetry reason we recall that

$$
\phi_{-}^{*}=-\phi_{+}, \phi_{+}^{*}=-\phi_{-},
$$

giving in particular from (4.7), (4.8),

$$
\begin{aligned}
& \phi-\phi\left(U_{0}\right)+\phi_{+}^{*} \backsim \operatorname{dist}\left(x, \pi_{x}\left(K_{-}\right)\right)^{2}, \\
& \phi-\phi\left(U_{0}\right)+\phi_{+} \backsim \operatorname{dist}\left(x, \pi_{x}\left(K_{-}^{*}\right)\right)^{2} .
\end{aligned}
$$

Let $\mu_{1}^{*}$ be the eigenvalue of $P_{*}^{(0)}:=-\Delta_{t}^{(0)}$ that is $o(h)$. As before, this is also the eigenvalue $o(h)$ of $-\Delta_{t}^{(1)}$ and the corresponding eigenspaces $E_{*}^{(0)}$ and $E_{*}^{(1)}$ are respectively spanned by the eigenfunctions

$$
\begin{array}{r}
e_{0}^{*}(x ; h)=\chi_{0}(x) e^{-\frac{1}{h} \phi_{+}^{*}(x)} h^{-\frac{n}{4}} a_{0}^{*}(x ; h)+\mathcal{O}\left(e^{-S_{0} / h}\right) \\
\text { and } \quad e_{1}^{*}(x, h)=h^{-n / 4} c_{j}(h) e^{-\frac{1}{h}\left(\phi(x)-\phi\left(U_{1}\right)\right)} \chi_{1}(x)+\mathcal{O}\left(h^{-N_{1}} e^{-\frac{1}{h}\left(S_{1}-\epsilon_{0}\right)}\right)
\end{array}
$$

Now, using that our eigenvalues and operators are real, we know by duality that $\mu_{1}^{*}=\mu_{1}$, and that $\left(E_{*}^{(0)}, E^{(0)}\right)$ and $\left(E_{*}^{(1)}, E^{(1)}\right)$ are dual pairs for the scalar products $(u \mid v)_{L^{2}}$ and $(u \mid v)_{A}$ respectively. Following [12, Subsection 10.3] we know that $\left(a_{0,0}^{*}\left(U_{0}\right) \mid a_{0,0}\left(U_{0}\right)\right)_{A} \neq 0$ and that $e_{0}^{*}$ can be normalized so that

$$
\left(e_{0}^{*} \mid e_{0}\right)_{A}=1 \text {. }
$$


Similarly, denoting by $e_{1}^{*}$ the $L^{2}$ normalized eigenfuncion spanning $E_{*}^{(0)}$, we have from (4.5.4.11)

$$
\left(e_{1}^{*} \mid e_{1}\right)=1+\mathcal{O}\left(e^{-\frac{1}{C h}}\right) .
$$

Let $\left(\lambda_{1}\right)$ be the (scalar) matrix of $d_{\phi}: E^{(0)} \rightarrow E^{(1)}$ with respect to the bases $\left(e_{1}\right)$ and $\left(e_{0}\right)$. Let also $\left(\lambda_{1}^{*}\right)$ be the (scalar valued) matrix of $d_{\phi}^{A, *}$ for the same bases. The eigenvalue $\mu_{1}$ can be viewed as the scalar $d_{\phi}^{A, *} d_{\phi}: E^{(0)} \rightarrow E^{(0)}$. We get

$$
\mu_{1}=\lambda_{1}^{*} \lambda_{1}
$$

and

$$
\bar{\lambda}_{1}=\left(e_{0}^{*} \mid d_{\phi} e_{1}\right)_{A}, \quad \bar{\lambda}_{1}^{*}=\left(g_{1} \mid d_{\phi}^{A, *} e_{0}\right)_{A}, j= \pm 1,
$$

where $g_{1}=e_{1}^{*}\left(1+\mathcal{O}\left(e^{-\frac{1}{C h}}\right)\right)$ is the vector in $E_{*}^{(0)}$ that is dual to $e_{1}$. Here the complex conjugate signs are superfluous since we work with real operators, eigenvalues and functions.

We skip the computation of $\lambda_{1}$, which is exactly the same as in [12], just recalling that the main term is equal to

$$
-c_{1}(h) h^{1-\frac{n}{2}} \int \chi_{1}(x)\left\langle A(x) a_{0}^{*}(x ; h) \mid d \chi(x)\right\rangle e^{-\frac{1}{h}\left(\phi_{+}^{*}(x)+\phi(x)-\phi\left(U_{1}\right)\right)} d x .
$$

and can be evaluated thanks to the stationary phase using (4.10). We get

$$
\lambda_{1}=h^{\frac{1}{2}} \ell_{1}(h) e^{-\frac{1}{h} S_{1}}\left(1+\mathcal{O}\left(e^{-\frac{1}{C h}}\right)\right), \quad \ell_{1} \sim \ell_{1,0}+h \ell_{1,1}+\ldots, \quad \ell_{1,0} \neq 0 .
$$

similarly, $\lambda_{1}^{*}$ ca be evaluated in a dual point of view as in [12] and we also get

$$
\lambda_{1}^{*}=h^{\frac{1}{2}} \ell_{1}^{*}(h) e^{-\frac{1}{h} S_{1}}\left(1+\mathcal{O}\left(e^{-\frac{1}{C h}}\right)\right), \quad \ell_{1}^{*}(h) \sim \ell_{1,0}^{*}+h \ell_{1,1}^{*}+\ldots, \quad \ell_{1,0}^{*} \neq 0 .
$$

We eventually claim that $\ell_{1,0} \ell_{1,0}^{*}>0$. Indeed, this number is real and different form zero and if we deform our matrices to reach the selfadjoint case (with $A>0$ ) we see that we have a positive sign). Combining this with (4.14), the proof of Theorem 4.1 is complete.

\section{Some models of KFP type operators}

\subsection{Probabilistic description}

Here we shall give some examples of Kramers-Fokker-Planck type operators. We begin with a very short review of stochastic calculus in order to explain their probabilistic origin, and refer to the books [17], [18] for more details. Part of this material 
can be also found in [7], [5] and [6], from where the example of the chain of anharmonic oscillators is taken.

Let $x(t) \in \mathbb{R}^{n}$ be a stochastic process satisfying the following stochastic differential equation

$$
d x(t)=b(x(t)) d t+\sigma d w,
$$

where $w$ is the $m$-dimensional Wiener process, $\sigma$ is a linear map from $\mathbb{R}^{m}$ to $\mathbb{R}^{n}$, and $b$ is a $C^{\infty}$-vector field on $\mathbb{R}^{n}$, all of whose derivatives are bounded. Under these assumptions, there exists a unique global solution $x(t)$ of (5.1), for a given initial data $x(0)=x$, independent of $w$, in an adapted stochastic $L^{2}$ setting - see the references already mentioned. Then we can define a semigroup of operators $T^{t}, t \geq 0$, by

$$
\mathbb{E}\left(\varphi(x(t)) \mid \mathcal{F}^{s}\right)=T^{t-s} \varphi(x(s)), \quad \text { a.s. }
$$

when $0 \leq s \leq t$. Here $\mathcal{F}^{t}$ is the filtration associated to $\{w(s)-w(0) ; 0 \leq s \leq t\}$ and $x$, and $\varphi \in C_{(0)}\left(\mathbb{R}^{n}\right)$, where $C_{(0)}\left(\mathbb{R}^{n}\right)$ is the Banach space of continuous functions vanishing at infinity, with the topology of the uniform convergence. Then $T^{t}$ is a strongly continuous positivity preserving contraction semigroup, whose infinitesimal generator is given on $C_{0}^{\infty}\left(\mathbb{R}^{n}\right)$ by

$$
L=\nabla \cdot D \nabla+b(x) \cdot \nabla
$$

where $D=\frac{1}{2} \sigma \sigma^{t}$. The idea now is to extend $T^{t}$ to a larger class of test functions, and then to study the evolution of the adjoint $\left(T^{t}\right)^{*}$ on the dual space of bounded measures. To be precise, let us denote by $d \mu_{t}(x)$ the probability distribution for $x(t)$, defined for all $t \geq 0$. We then have

$$
\mathbb{E}(\varphi(x(t)))=\int \varphi(x) d \mu_{t}(x), \quad \varphi \in C_{(0)}\left(\mathbb{R}^{n}\right),
$$

and we get by (5.2) that $\mu_{t}=\left(T^{t}\right)^{*} \mu_{0}$, where $\left(T^{t}\right)^{*}$ is the adjoint of the operator $T^{t}$ acting on the Banach space of bounded measures on $\mathbb{R}^{n}$.

We shall now extend the space of test functions. When doing so, we introduce the Hilbert space

$$
\mathcal{H}=L^{2}\left(\mathbb{R}^{n}, e^{-\Phi(x)} d x\right)
$$

where $\Phi \in C^{\infty}\left(\mathbb{R}^{n}\right)$. We shall make the following assumptions concerning $\Phi$ :

$$
\begin{gathered}
\partial_{x}^{\alpha} \Phi(x)=\mathcal{O}(1), \quad|\alpha| \geq 2 \\
\frac{1}{2} b(x) \cdot \nabla \Phi(x)+\frac{1}{4}\left\langle D \nabla_{x} \Phi, \nabla_{x} \Phi\right\rangle-\frac{1}{2} \operatorname{div} b \leq \mathcal{O}(1)
\end{gathered}
$$


and

$$
\partial_{x}^{\alpha}\left(\frac{1}{2} b(x) \cdot \nabla \Phi(x)+\frac{1}{4}\left\langle D \nabla_{x} \Phi, \nabla_{x} \Phi\right\rangle-\frac{1}{2} \operatorname{div} b\right)=\mathcal{O}(1), \quad|\alpha| \geq 2 .
$$

These conditions will be fulfilled in the case that we shall study in what follows, since in the supersymmetric case it is straightforward to verify that they are equivalent to (3.4). Now we can identify the dual $\mathcal{H}^{\prime}$ of $\mathcal{H}$ with the space of densities

$$
\mathcal{H}^{*}=L^{2}\left(\mathbb{R}^{n}, e^{\Phi(x)} d x\right) .
$$

Assume that the measures $d \mu_{t}$ are absolutely continuous with density in $\mathcal{H}^{*}$, and write

$$
d \mu_{t}=f(t, .) d x
$$

identifying the measure $d \mu_{t}$ with the corresponding density $f_{t}$. We denote again by $\left(T^{t}\right)^{*}$ acting on $\mathcal{H}^{*}$ the adjoint of $T^{t}$ acting on $\mathcal{H}$. We introduce the formal adjoint operator $L^{*}$ on $\mathcal{H}^{*}$ of $L$ on $\mathcal{H}$, with the domain $C_{0}^{\infty}\left(\mathbb{R}^{n}\right)$, which is given by

$$
L^{*}=\nabla \cdot D \nabla-\nabla \cdot b(x) .
$$

We have the following result.

Lemma 5.1 Assume that $\Phi \in C^{\infty}\left(\mathbb{R}^{n}\right)$ satisfies (5.4), (5.6). Then operator $-L$ on $\mathcal{H}$ (resp. $-L^{*}$ on $\left.\mathcal{H}^{*}\right)$ is m-accretive, and $T^{t}$ (resp $\left.\left(T^{t}\right)^{*}\right)$ is a strongly continuous semigroups on $\mathcal{H}$ (resp. $\left.\mathcal{H}^{*}\right)$, with infinitesimal generators given by $L$ (resp. $\left.L^{*}\right)$.

Proof. It will be more convenient to work in the unweighted space $L^{2}\left(\mathbb{R}^{n}\right)$. To this end, if $\phi \in \mathcal{H}$, we write $\phi(x)=e^{\Phi(x) / 2} \psi(x), \psi \in L^{2}$. If

$$
\partial_{t} \phi=L \phi
$$

then the equation satisfied by $\psi$ is

$$
\partial_{t} \psi=\left(e^{-\Phi / 2} L e^{\Phi / 2}\right) \psi
$$

so that

$$
\partial_{t} \psi=\left(\partial_{x}+\partial_{x} \Phi / 2\right) \cdot D\left(\partial_{x}+\partial_{x} \Phi / 2\right) \psi+b(x) \cdot\left(\partial_{x}+\partial_{x} \Phi / 2\right) \psi
$$

Let $C$ be a sufficiently large constant. According to (5.4+5.6), the operator

$$
-\left(\left(\partial_{x}+\partial_{x} \Phi / 2\right) \cdot D\left(\partial_{x}+\partial_{x} \Phi / 2\right)+b(x) \cdot\left(\partial_{x}+\partial_{x} \Phi / 2\right)\right)+C
$$


has a symbol satisfying the hypotheses (2.4)-(2.7). Here we may recall that the vector field $b$ is bounded on $\mathbb{R}^{n}$ together with all of its derivatives. An application of Corollary 3.2 in [12] shows that its maximal closed realization in $L^{2}$ coincides with the graph closure on $\mathcal{S}$. Coming back to $\mathcal{H}$ and denoting by $L$ again its maximal closed extension, we get that $L+C$ is maximal accretive, and that $T^{t}$ is a strongly continuous semigroup, thanks to the Hille-Yosida Theorem. As for the dual semigroup on $\mathcal{H}^{*}$, we also get (for example, using Corollary 10.6 in [19]), that the same occurs for $\left(T^{t}\right)^{*}$ and $L^{*}$. The proof is complete.

From the preceding discussion, we get the equation satisfied by the density $f$ for an initial data $f_{0} \in \mathcal{H}^{*}$,

$$
\left\{\begin{array} { l } 
{ \partial _ { t } f + ( - L ^ { * } ) f = 0 } \\
{ f | _ { t = 0 } = f _ { 0 } }
\end{array} \quad \text { i.e. } \quad \left\{\begin{array}{l}
\partial_{t} f+(-\nabla \cdot D \nabla+\nabla \cdot b) f=0 \\
\left.f\right|_{t=0}=f_{0}
\end{array}\right.\right.
$$

where we recall that $D=\sigma \sigma^{t} / 2$. In particular we have $d \mu_{t}=f(t,) d x=.\left(T^{t}\right)^{*} \mu_{0}$ in the space of bounded measures.

If there exists an invariant probability measure $\mu_{\infty}$, then its density $\mathcal{M}$ is in $\mathcal{H}^{*}$. In our present study we shall essentially make the choice $C^{-1} e^{-\Phi}=\mathcal{M}$, but there are cases (see e.g. 17], [5]), where it may happen that no invariant measure is known, and that another choice of the function $\Phi$ is necessary. Such a function $\mathcal{M}$ will be called a Maxwellian of the process. Notice that if it exists, it is a 0 -eigenfunction of $L^{*}$ and positive.

Remark 5.2. Notice that it may also happen that there exists an invariant measure, which fails to be finite. We also associated to it a function that we will call again Maxwellian (and denote again by $\mathcal{M}$ ). In that case of course it cannot be normalized.

Equation (5.7) is nearly the Kramers-Fokker-Planck type equation that we studied in the first part of the paper. In the following sections we shall also do the following two things: first we shall exhibit the semiclassical scaling, which corresponds to the low temperature limit in the models we are going to study later. Second, we shall change our unknown by posing $f=e^{-\Phi(x) / 2} u$ (forgetting for a while the semiclassical scaling), in order to work in the flat space $L^{2}$ rather than in $\mathcal{H}$. Finally, in the three models that we present in the next subsections (Witten, Kramers-Fokker-Planck, and the chain of anharmonic oscillators), we shall recognize the supersymmetric structure. 


\subsection{Witten and Kramers-Fokker-Planck operators}

We begin with the Witten case. It corresponds to an evolution equation with a gradient field $-\gamma \nabla V(x)$ and a diffusion force coming from a heat bath at a temperature $T$. We have

$$
d x=-\gamma \partial_{x} V d t+\sqrt{2 \gamma T} d w .
$$

Here $x \in \mathbb{R}^{n}$ is the spatial variable, the parameter $\gamma$ is a friction coefficient, and $w$ is an $n$-dimensional Wiener process of mean 0 and variance 1 . With the notation of the preceding subsection, we recover an equation of type (5.1) with $D=\sigma \sigma^{t} / 2=\gamma T I_{d}$ and $b(x)=-\gamma \partial_{x} V$. Equation (5.7) for the density in this case is then

$$
\left\{\begin{array} { l } 
{ \partial _ { t } f - \gamma T \partial _ { x } ^ { 2 } f - \gamma \partial _ { x } ( \partial _ { x } V f ) = 0 } \\
{ f | _ { t = 0 } = f _ { 0 } }
\end{array} \quad \text { i.e. } \quad \left\{\begin{array}{l}
\partial_{t} f-\gamma \partial_{x}\left(T \partial_{x}+\partial_{x} V\right) f=0 \\
\left.f\right|_{t=0}=f_{0}
\end{array}\right.\right.
$$

Posing $T=h / 2$ and multiplying by $h$ gives the semiclassical equation

$$
h \partial_{t} f-\frac{\gamma}{2} h \partial_{x}\left(h \partial_{x}+2 \partial_{x} V\right) f=0 .
$$

It is then clear that an associated Maxwellian of the process is

$$
\mathcal{M}(x)=e^{-2 V(x) / h} .
$$

Writing $f=\mathcal{M}^{1 / 2} u$, we obtain from (5.9) that

$$
h \partial_{t} u+\frac{\gamma}{2}\left(-h \partial_{x}+\partial_{x} V\right)\left(h \partial_{x}+\partial_{x} V\right) u=0
$$

Here we recognize the Witten operator $W=\left(-h \partial_{x}+\partial_{x} V\right)\left(h \partial_{x}+\partial_{x} V\right)$. In the notation of (3.1) $-(3.5)$, it corresponds to a supersymmetric operator with

$$
A=\frac{\gamma}{2} I_{d}, \quad \phi(x)=V(x)
$$

Assumptions of type (3.4) on $V$ are then fulfilled if

$$
\partial^{\alpha} V(x)=\left\{\begin{array}{l}
\mathcal{O}(1) \quad \text { when } \quad|\alpha|=2 \\
\mathcal{O}\left(\langle x\rangle^{-1}\right) \quad \text { when }|\alpha| \geq 3
\end{array}\right.
$$

If we also suppose that $V$ is a Morse function with two local minima and a saddle point of index one, such that

$$
|\nabla V| \geq 1 / C, \quad \text { for } \quad|x| \geq C>0
$$


then the dynamical asumptions (2.14) $-(\underline{2.16})$ are satisfied (we skip the proof here, which will be given later in the more complex case of the chain of oscillators). In particular, in this case we get Theorem 3.2. Of course, the corresponding result follows also in the case of a single well and the sea, i.e. when $V$ has precisely one local minimum and a saddle point (Theorem 4.1 and the remark following it).

We proceed now to discuss the Kramers-Fokker-Planck case, and follow the same method. The stochastic equation of type (5.1) comes here from the Newton law

$$
\left\{\begin{array}{l}
d x=y d t \\
d y=-\gamma y d t-\partial_{x} V(x) d t+\sqrt{2 \gamma T} d w
\end{array} .\right.
$$

The parameter $\gamma$ is a friction coefficient, and the particle of position $x \in \mathbb{R}^{n}$ and velocity $y \in \mathbb{R}^{n}$ is submitted to an external force field derived from a potential $V$, with $w$ being an $n$-dimensional Brownian process of mean 0 and variance 1 . With the notation of the preceding subsection, we therefore have

$$
D=\sigma \sigma^{t} / 2=\left(\begin{array}{cc}
0 & 0 \\
0 & \gamma T I_{d}
\end{array}\right) \quad \text { and } \quad b(x, y)=\left(\begin{array}{c}
y \\
-\gamma y-\partial_{x} V
\end{array}\right)
$$

The corresponding equation for the density (5.7) is then

$$
\left\{\begin{array}{l}
\partial_{t} f-\gamma T \Delta_{y} f+\partial_{x}(y f)+\partial_{y}\left(-\gamma y f-\partial_{x} V f\right)=0 \\
\left.f\right|_{t=0}=f_{0}
\end{array}\right.
$$

i.e.

$$
\left\{\begin{array}{l}
\partial_{t} f-\gamma \partial_{y} \cdot\left(T \partial_{y}+y\right) f+y \partial_{x} f-\partial_{x} V \partial_{y} f=0 \\
\left.f\right|_{t=0}=f_{0}
\end{array}\right.
$$

Posing $T=h / 2$ and multiplying by $h$ gives the semiclassical formulation

$$
h \partial_{t} f-\frac{\gamma}{2} h \partial_{y} \cdot\left(h \partial_{y}+2 y\right) f+y h \partial_{x} f-\partial_{x} V h \partial_{y} f=0 .
$$

A Maxwellian of the process is then

$$
\mathcal{M}(x, y)=C^{-1} e^{-2\left(V(x)+y^{2} / 2\right) / h}
$$

where $C$ is a normalization constant. If we write $f=\mathcal{M}^{1 / 2} u$, then (5.15) gives

$$
h \partial_{t} u+\frac{\gamma}{2}\left(-h \partial_{y}+y\right) \cdot\left(h \partial_{y}+y\right) u+\gamma y \cdot h \partial_{x} u-\partial_{x} V \cdot h \partial_{y} u=0
$$


This is the semiclassical Kramers-Fokker-Planck operator (1.1) that we studied in [11], [12], and the first part of the present paper. In the notation of section 3, it corresponds to a supersymmetric operator with

$$
A=\frac{1}{2}\left(\begin{array}{cc}
0 & I_{d} \\
-I_{d} & \gamma
\end{array}\right) \quad \text { and } \quad \phi(x, y)=V(x)+y^{2} / 2
$$

The assumptions (3.4) are fulfilled if

$$
\partial^{\alpha} V(x)=\mathcal{O}(1) \quad \text { when } \quad|\alpha| \geq 2
$$

As in the Witten case, if we also suppose that $V$ is a Morse function with precisely two local minima and a saddle point of index one, and that

$$
|\nabla V| \geq 1 / C, \quad \text { for } \quad|x| \geq C>0
$$

then the dynamical asumptions (2.14) -(2.16) are satisfied, and Theorem 3.2 is applicable. The corresponding result in the case of a single well and the sea, i.e. when $V$ has one local minimum and a saddle point, is also valid (Theorem 4.1 and the following remark).

\section{Chains of anharmonic oscillators}

The last example that we give comes from the series of papers [7], [5] [6]. It is a model describing a chain of two anharmonic oscillators coupled with two heat baths at each side.

The particles are described by their respective position and velocity $\left(x_{j}, y_{j}\right) \in \mathbb{R}^{2 d}$. We suppose that for each oscillator $j \in\{1,2\}$, the particles are submitted to an external force derived from a potential $V_{j}\left(x_{j}\right)$, and that there is a coupling between the two oscillators derived from a potential $V_{c}\left(x_{2}-x_{1}\right)$. We denote by $V$ the sum

$$
V(x)=V_{1}\left(x_{1}\right)+V_{2}\left(x_{2}\right)+V_{c}\left(x_{2}-x_{1}\right),
$$

where $x=\left(x_{1}, x_{2}\right)$, and we also write $y=\left(y_{1}, y_{2}\right)$. By $z_{j}, j \in\{1,2\}$ we shall denote the variables describing the state of the particles in each of the heat baths, and set $z=\left(z_{1}, z_{2}\right)$. We suppose that the particles in each bath are submitted to a coupling with the nearest oscillator, a friction force and a thermal diffusion at temperature $T_{j},(j=1,2)$. We denote by $w_{j}, j \in\{1,2\}$, two $d$-dimensional brownian motions of mean 0 and variance 1 , and set $w=\left(w_{1}, w_{2}\right)$. 


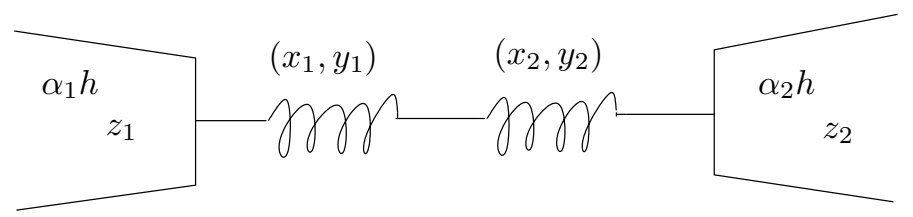

Figure 2: Oscillators coupled to heat baths

The fundamental system of equations of type (5.1) is then written as follows, (see 7] for more detail concerning the physical constants)

$$
\left\{\begin{array}{l}
d x_{1}=y_{1} d t \\
d y_{1}=-\partial_{x_{1}} V(x) d t+z_{1} d t \\
d z_{1}=-\gamma z_{1} d t+\gamma x_{1} d t-\sqrt{2 \gamma T_{1}} d w_{1} \\
d z_{2}=-\gamma z_{1} d t+\gamma x_{2} d t-\sqrt{2 \gamma T_{2}} d w_{2} \\
d y_{2}=-\partial_{x_{2}} V(x) d t+z_{2} d t \\
d x_{2}=y_{2} d t
\end{array}\right.
$$

The parameter $\gamma$ is the friction coefficient in the baths. In the $(x, y, z)$ variables, the diffusion matrix and the drift appearing in (5.1) are therefore

$$
D=\sigma \sigma^{t} / 2=\left(\begin{array}{ccc}
0 & 0 & 0 \\
0 & 0 & 0 \\
0 & 0 & \gamma T I_{d}
\end{array}\right) \quad \text { and } \quad b(x)=\left(\begin{array}{c}
y \\
-\partial_{x} V+z \\
\gamma(x-z)
\end{array}\right) .
$$

(for simplicity we identified $T$ with the $2 \mathrm{~d}$ times $2 \mathrm{~d}$ diagonal matrix with coefficients $T_{1} I$ and $T_{2} I$. The corresponding equation (5.7) for the density is then

$$
\left\{\begin{array}{l}
\partial_{t} f-\gamma T \partial_{z}^{2} f+\partial_{x}(y f)+\partial_{y}\left(-\partial_{x} V f+z f\right)+\partial_{z}(\gamma(x-z))=0 \\
f_{t=0}=f_{0}
\end{array}\right.
$$

where $T \partial_{z}^{2}$ stands for $T_{1} \partial_{z_{1}}^{2}+T_{2} \partial_{z_{2}}^{2}$. We get

$$
\left\{\begin{array}{l}
\partial_{t} f-\gamma \partial_{z}\left(T \partial_{z}+(z-x)\right) f+y \partial_{x} f-\left(\partial_{x} V-z\right) \partial_{y} f=0 \\
f_{t=0}=f_{0}
\end{array}\right.
$$

Notice that it is very close to the Witten and Kramers-Fokker-Planck operator. For a semiclassical formulation, we pose $T_{1}=\alpha_{1} h / 2$ and $T_{2}=\alpha_{2} h / 2$, and we multiply (6.3) by $h$. This gives

$$
\begin{aligned}
h \partial_{t} f & +\frac{\gamma}{2} \alpha_{1}\left(-h \partial_{z_{1}}\right)\left(h \partial_{z_{1}}+2\left(z_{1}-x_{1}\right) / \alpha_{1}\right) f \\
& +\frac{\gamma}{2} \alpha_{2}\left(-h \partial_{z_{2}}\right)\left(h \partial_{z_{2}}+2\left(z_{2}-x_{2}\right) / \alpha_{2}\right) f \\
& +\left(y h \partial_{x} f-\left(\partial_{x} V-z\right) h \partial_{y}\right) f=0 .
\end{aligned}
$$


At this stage it is difficult to exhibit a Maxwellian. Indeed the existence of an invariant measure is a difficult problem solved in some particular case in [7]. Anyway, it is clear that the function

$$
\Phi(x, y, z)=V(x)+y^{2} / 2+z^{2} / 2-z x
$$

plays a special role, in fact it is the classical energy at temperature 1. We can also check that in the case of same temperatures $\left(\alpha_{1}=\alpha_{2} \stackrel{\text { def }}{=} \alpha\right)$, the function

$$
\mathcal{M}_{\alpha}=C^{-1} e^{-2 \Phi / \alpha h}
$$

is a Maxwellian of the process. We use this function to define the weighted space $\mathcal{H} \stackrel{\text { def }}{=} L^{2}\left(e^{-2 \Phi / \alpha h} d x d y d z\right)$ as in (5.3) $)$ and in order to work in the flat space $L^{2}$ we make the change of unknown

$$
f=\mathcal{M}_{\alpha}^{1 / 2} u
$$

Equation (5.14) becomes

$$
\begin{aligned}
h \partial_{t} u & +\frac{\gamma}{2} \alpha_{1}\left(-h \partial_{z_{1}}+\frac{1}{\alpha}\left(z_{1}-x_{1}\right)\right)\left(h \partial_{z_{1}}+\left(\frac{2}{\alpha_{1}}-\frac{1}{\alpha}\right)\left(z_{1}-x_{1}\right)\right) u \\
& +\frac{\gamma}{2} \alpha_{2}\left(-h \partial_{z_{2}}+\frac{1}{\alpha}\left(z_{2}-x_{2}\right)\right)\left(h \partial_{z_{2}}+\left(\frac{2}{\alpha_{2}}-\frac{1}{\alpha}\right)\left(z_{2}-x_{2}\right)\right) u \\
& +\left(y h \partial_{x}-\left(\partial_{x} V-z\right) h \partial_{y}\right) u=0 .
\end{aligned}
$$

We impose the following condition on the parameter $\alpha$ :

$$
\alpha \geq \max \left\{\alpha_{1}, \alpha_{2}\right\} / 2 \text {. }
$$

which corresponds to a semiclassical study at "reference" temperature $\alpha h / 2$ not too low.

Unfortunately we are not able to find any supersymmetric structure in the case of different temperatures, since a Maxwellian is not known in this case. From now on we therefore stick to the case of identical temperatures $T=h / 2$ so that

$$
\alpha=\alpha_{1}=\alpha_{2}=1
$$

Equation (6.5) becomes

$$
\begin{aligned}
h \partial_{t} u & +\frac{\gamma}{2}\left(-h \partial_{z_{1}}+\left(z_{1}-x_{1}\right)\right)\left(h \partial_{z_{1}}+\left(z_{1}-x_{1}\right)\right) u \\
& +\frac{\gamma}{2}\left(-h \partial_{z_{2}}+\left(z_{2}-x_{2}\right)\right)\left(h \partial_{z_{2}}+\left(z_{2}-x_{2}\right)\right) u \\
& +\left(y h \partial_{x}-\left(\partial_{x} V-z\right) h \partial_{y}\right) u=0
\end{aligned}
$$


and the Maxwellian was already exhibited $\mathcal{M}_{1}=C^{-1} e^{-2 \Phi / h}$. This equation can be written $h \partial_{y} u+P u=0$ where

$$
\begin{aligned}
P= & \frac{\gamma}{2}\left(-h \partial_{z_{1}}+\left(z_{1}-x_{1}\right)\right)\left(h \partial_{z_{1}}+\left(z_{1}-x_{1}\right)\right) \\
& +\frac{\gamma}{2}\left(-h \partial_{z_{2}}+\left(z_{2}-x_{2}\right)\right)\left(h \partial_{z_{2}}+\left(z_{2}-x_{2}\right)\right)+\left(y h \partial_{x}-\left(\partial_{x} V-z\right) h \partial_{y}\right) .
\end{aligned}
$$

In the notations of Section $3(3.1+3.5)$, we can write $P$ as a Witten-Hodge laplacian $P=-\Delta_{A}^{(0)}$ with a supersymmetric phase $\phi$ given by

$$
\phi \stackrel{\text { def }}{=} \Phi=V(x)+y^{2} / 2+z^{2} / 2-z x,
$$

and the non-degenerate matrix $A=B+C$ given by

$$
A=\frac{1}{2}\left(\begin{array}{ccc}
0 & I_{d} & 0 \\
-I_{d} & 0 & 0 \\
0 & 0 & \gamma I_{d}
\end{array}\right) \quad \text { with } B=\frac{1}{2}\left(\begin{array}{ccc}
0 & 0 & 0 \\
0 & 0 & 0 \\
0 & 0 & \gamma I_{d}
\end{array}\right), \quad C=\frac{1}{2}\left(\begin{array}{ccc}
0 & I_{d} & 0 \\
-I_{d} & 0 & 0 \\
0 & 0 & 0
\end{array}\right) \text {. }
$$

In order to complete the semiclassical study as in [12, we only need additional conditions on the potentials $V_{1}, V_{2}$ and $V_{c}$. It is clear that the conditions

$$
\partial^{\alpha} V_{\varepsilon}(x)=\mathcal{O}(1) \quad \text { when } \quad|\alpha| \geq 2, \quad \text { with } \varepsilon=1,2 \text { and } c
$$

imply (3.4). In view of the definition (6.8), it is straightforward that $\phi$ has exactly the same number of critical points than $V(x)-x^{2} / 2$ with same index. For this it is sufficient to notice that there is a natural splitting of the variables for $\Phi$ given by

$$
\Phi=\left(V(x)-x^{2} / 2\right)+y^{2} / 2+(z-x)^{2} / 2 .
$$

We postpone to the end of this section the proof of the following lemma:

Lemma 6.1 Suppose that $V$ satisfies (6.9). If in addition $V(x)-x^{2} / 2$ is a Morse function and there exists $C$ such that

$$
|\partial V(x)-x| \geq 1 / C \text { when }|x| \geq C
$$

then (3.7) and the dynamical conditions (2.14,2.16) are fulfilled.

As a consequence we can apply Theorem 3.2 to operator $P$ : 
Proposition 6.2 Consider $P$ given by (6.7) and suppose that $V$ satisfies (6.9) and (6.10). Then if the effective potential $V(x)-x^{2} / 2$ is of double well type, (two local minima and a saddle point of index 1), the hypotheses of Theorem 3.2 are fulfilled and as a consequence its conclusions apply to operator $P$.

Proof. It is straightforward. From the construction of $P$, hypotheses (3.1) 3.3) are fulfilled. From (6.9), hypothesis (3.4) is satisfied. Since the effective potential $V(x)-x^{2} / 2$ is a morse function of double well type, then (3.8) is also satisfied since, as already noticed, $V(x)-x^{2} / 2$ and $\phi$ have the same number of critical point with same index. Eventually using Lemma 6.1 and (6.10) we get that hypothese (3.7) and the dynamical conditions (2.14 2.16) are fulfilled. The proof is complete and the conclusions of Theorem 3.2 apply to $P$.

Remark 6.3. Of course the corresponding result follows in the case of a well and the sea, ie when $V(x)-x^{2} / 2$ has one minimum and a saddle point (Theorem4.1 and the remark after).

A simple family of such potentials is given for example by the ones for which $V_{1}\left(x_{1}\right)-x_{1}^{2} / 2$ of double well type, $V_{2}\left(x_{2}\right)-x_{2}^{2} / 2$ of single well type, and $V_{c}$ sufficiently small. Here is an example of such potentials in $1 \mathrm{~d}$ :

$$
V_{1}\left(x_{1}\right)=x_{1}^{2} / 2+5 \sqrt{\left(x_{1}^{2}-1\right)^{2}+1}, \quad V_{2}\left(x_{2}\right)=5 x_{2}^{2}, \quad V_{c}\left(x^{\prime}\right)=\frac{1}{10} \cos \left(x^{\prime}\right) .
$$

Here $x_{1}, x_{2}, x^{\prime} \in \mathbb{R}$.

Remark 6.4. We did all the computations in the case of 2 oscillators. It is clear that the preceding supersymmetric construction works as well in the case of $N$ oscillators coupled and with two heat bathes at each side as in [7]. We did not try to verify the dynamical conditions in these cases. Eventually recall that the complete study at different temperatures seems difficult to treat (see e.g. the recent work by Hairer and Mattingly [9] in the case of 3 oscillators).

\section{Proof of Lemma 6.1.}

We only prove here condition (3.7) and (2.16) since the proof of other ones follow the same kind of arguments. The proof uses strongly the fact that the symbols are with quadratic growth at most. We write $p=p_{2}+i p_{1}+p_{0}$ for the symbol of 
the corresponding Hodge Laplacian on 0 -forms (minus the constant $\gamma h d / 2$ ) where denoting $(\xi, \eta, \zeta)$ the dual variables of $(x, y, z)$ we have

$$
p_{2}=\frac{\gamma}{2} \zeta^{2}, \quad p_{1}=y \xi-\left(\partial_{x} V-z\right) \eta, \quad p_{0}=\frac{\gamma}{2}(z-x)^{2} .
$$

In particular, with the notations of Section 2, we have

$$
\nu\left(x, y, z, \partial_{x}, \partial_{y}, \partial_{z}\right)=y \partial_{x}-\left(\partial_{x} V-z\right) \partial_{y}
$$

We denote by $x_{1}, \ldots, x_{N}$ the critical points of $V(x)-x^{2} / 2$, and notice that the critical points of $\Phi$ are $\left(x_{j}, 0, x_{j}\right)$ for $j=1, \ldots, N$. According to definitions (2.11) and (6.11), the critical set $\mathcal{C}$ of $p$ is made of the points $\rho_{j}=\left(x_{j}, 0, x_{j}, 0,0,0\right)$ for $j=1, \ldots, N$. We also introduce $\pi_{x}$ (resp. $\pi_{x y z}$ ) the orthogonal projections on $\mathbb{R}_{x}^{d}$ (resp. $\mathbb{R}_{x, y, z}^{3 d}$ ) from $\mathbb{R}^{6 d}$.

Let now $\varepsilon>0$ be a fixed constant. Since $V(x)-x^{2} / 2$ is a Morse function, it is non-degenerate, so that with (6.10) we get the following: there exists $C_{\varepsilon}$ such that

$$
\forall x \notin \pi_{x}(\mathcal{C}+B(0, \varepsilon)), \quad|\partial V(x)-x| \geq 1 / C_{\varepsilon}
$$

From this result we get easily the following one: there exists $C_{\varepsilon}^{\prime}$ such that

$$
\forall(x, y, z) \notin \pi_{x, y, z}(\mathcal{C}+B(0, \varepsilon)), \quad|\partial V(x)-x|+|z-x|+|y| \geq 1 / C_{\varepsilon}^{\prime} .
$$

This proves (3.7).

In order to complete the proof of (2.16) we study in detail the characteristics of the flow generated by the vector field $\nu\left(x, y, z, \partial_{x}, \partial_{y}, \partial_{z}\right)$. We first notice that the flow is complete since the gradient of $\Phi$ is Lipschitz. Let $\left(x_{0}, y_{0}, z_{0}\right) \notin \pi_{x, y, z}(\mathcal{C}+B(0, \varepsilon))$. We denote by $(x(t), y(t), z(t))$ the integral curves of $\nu$ for $t \in \mathbb{R}$ with $x(0)=x_{0}$, $y(0)=y_{0}, z(0)=z_{0}$ and

$$
\left\{\begin{array}{l}
\dot{x}=y \\
\dot{y}=-\left(\partial_{x} V(x)-z\right) \\
\dot{z}=0
\end{array}\right.
$$

and we have to study

$$
p_{0}\left(\exp (t \nu)\left(x_{0}, y_{0}, z_{0}\right)\right)=\frac{\gamma}{2}|x(t)-z(t)|^{2}=\frac{\gamma}{2}\left|x(t)-z_{0}\right|^{2}
$$

We split the study into two cases. Let $t \in[0,1]$. 
First case: Suppose $\left|y_{0}\right| \geq\left|\partial_{x} V\left(x_{0}\right)-z_{0}\right|$. We write that

$$
\begin{aligned}
y(t) & =y_{0}+\int_{0}^{t} \dot{y}(s) d s \\
& =y_{0}-\int_{0}^{t}\left(\partial_{x} V(x(s))-z_{0}\right) d s \\
& =y_{0}-t\left(\partial_{x} V\left(x_{0}\right)-z_{0}\right)-\int_{0}^{t}\left(\int_{0}^{s} V^{\prime \prime}(x(r)) \dot{x}(r) d r\right) d s \\
& =y_{0}-t\left(\partial_{x} V\left(x_{0}\right)-z_{0}\right)-\int_{0}^{t}\left(\int_{0}^{s} V^{\prime \prime}(x(r)) y(r) d r\right) d s .
\end{aligned}
$$

Since $V^{\prime \prime}$ is uniformly bounded, and denoting by $C_{V} \geq 1$ a corresponding bound we get

$$
\sup _{s \in[0, t]}\left|y(s)-y_{0}\right| \leq t\left|\partial_{x} V\left(x_{0}\right)-z_{0}\right|+\frac{t^{2}}{2} C_{V} \sup _{s \in[0, t]}|y(s)| .
$$

This implies that on $\left[0, t_{V}\right]$ with $t_{V} \leq 1 / C_{V} \leq 1 / C_{V}^{1 / 2}$ we have

$$
\frac{1}{2} \sup _{s \in[0, t]}\left|y(s)-y_{0}\right| \leq t\left|\partial_{x} V\left(x_{0}\right)-z_{0}\right|+\frac{t^{2}}{2} C_{V}\left|y_{0}\right| .
$$

Since by assumption $\left|y_{0}\right| \geq\left|\partial_{x} V\left(x_{0}\right)-z_{0}\right|$ we get

$$
\sup _{s \in[0, t]}\left|y(s)-y_{0}\right| \leq 2 t\left|y_{0}\right|+t^{2} C_{V}\left|y_{0}\right| \leq 3 t\left|y_{0}\right| .
$$

We can then write that

$$
\begin{aligned}
x(t) & =x_{0}+\int_{0}^{t} \dot{x}(s) d s=x_{0}+\int_{0}^{t} y(s) d s \\
& =x_{0}+t y_{0}+\frac{3}{2} t^{2}\left|y_{0}\right| \phi(t)
\end{aligned}
$$

with $|\phi(t)| \leq 1$ on $\left[0, t_{V}\right]$. Recalling (6.14) and using the triangular inequality, we get for $t \in\left[0, t_{V}\right]$ that if $t_{V} \leq 1 / 4$

$$
\left|x(t)-z_{0}\right|^{2} \geq \max \left\{\left|x_{0}-z_{0}\right|-2 t\left|y_{0}\right|, \quad \frac{t}{2}\left|y_{0}\right|-\left|x_{0}-z_{0}\right|\right\}
$$

Let now $0<\theta<t_{V}$. We split again the study into two parts: 
1. If $\left|x_{0}-z_{0}\right| \geq \theta\left|y_{0}\right|$, we use the first expression in (6.17) and we get that

$$
\left|x(t)-z_{0}\right|^{2} \geq\left|x_{0}-z_{0}\right|(1-2 t / \theta) \geq \frac{1}{2}\left|x_{0}-z_{0}\right| \quad \text { on } \quad[0, \theta / 4] .
$$

2. If $\theta\left|y_{0}\right| \geq\left|x_{0}-z_{0}\right|$, we use the second expression in (6.17) and we get that

$$
\left|x(t)-z_{0}\right|^{2} \geq\left|y_{0}\right|(t / 2-\theta) \geq \frac{t_{V}}{8}\left|y_{0}\right| \quad \text { on } \quad\left[t_{V} / 2, t_{V}\right] .
$$

if $\theta \leq t_{V} / 8$.

In all cases we get that there exists a constant $c_{V}>0$ depending only on $C_{V}$ such that

$$
\left|x(t)-z_{0}\right|^{2} \geq c_{V} \max \left\{\left|y_{0}\right|,\left|x_{0}-z_{0}\right|,\left|\partial_{x} V\left(x_{0}\right)-z_{0}\right|\right\}
$$

on an interval of length at least $\theta$.

Second case: Suppose $\left|y_{0}\right| \leq\left|\partial_{x} V\left(x_{0}\right)-z_{0}\right|$. As in (6.15) we can write that

$$
y(t)=y_{0}-t\left(\partial_{x} V\left(x_{0}\right)-z_{0}\right)-\int_{0}^{t}\left(\int_{0}^{s} V^{\prime \prime}(x(r)) y(r) d r\right) d s .
$$

Since $V^{\prime \prime}$ is uniformly bounded, and denoting again by $C_{V} \geq 1$ a corresponding bound we get

$$
\sup _{s \in[0, t]}\left|y(s)-y_{0}+s\left(\partial_{x} V\left(x_{0}\right)-z_{0}\right)\right| \leq \frac{t^{2}}{2} C_{V} \sup _{s \in[0, t]}|y(s)|,
$$

so that

$$
\begin{aligned}
& \sup _{s \in[0, t]}\left|y(s)-y_{0}+s\left(\partial_{x} V\left(x_{0}\right)-z_{0}\right)\right| \\
& \leq \frac{t^{2}}{2} C_{V} \sup _{s \in[0, t]}\left|y(s)-y_{0}+s\left(\partial_{x} V\left(x_{0}\right)-z_{0}\right)\right|+\frac{t^{2}}{2} C_{V}\left(\left|y_{0}\right|+t\left|\partial_{x} V\left(x_{0}\right)-z_{0}\right|\right) .
\end{aligned}
$$

This implies that on $\left[0, t_{V}\right]$ with again $t_{V} \leq 1 / C_{V} \leq 1 / C_{V}^{1 / 2}$ we have

$$
\begin{aligned}
\frac{1}{2} \sup _{s \in[0, t]}\left|y(s)-y_{0}+s\left(\partial_{x} V\left(x_{0}\right)-z_{0}\right)\right| & \leq \frac{t^{2}}{2} C_{V}\left(\left|y_{0}\right|+t\left|\partial_{x} V\left(x_{0}\right)-z_{0}\right|\right) \\
& \leq C_{V} t^{2}\left|\partial_{x} V\left(x_{0}\right)-z_{0}\right|,
\end{aligned}
$$


since by assumption $\left|y_{0}\right| \leq\left|\partial_{x} V\left(x_{0}\right)-z_{0}\right|$. We therefore get

$$
\sup _{s \in[0, t]}\left|y(s)-y_{0}+s\left(\partial_{x} V\left(x_{0}\right)-z_{0}\right)\right| \leq 2 C_{V} t^{2}\left|\partial_{x} V\left(x_{0}\right)-z_{0}\right|
$$

We can then write that

$$
\begin{aligned}
x(t) & =x_{0}+\int_{0}^{t} \dot{x}(s) d s=x_{0}+\int_{0}^{t} y(s) d s \\
& =x_{0}+t y_{0}-\frac{t^{2}}{2}\left(\partial_{x} V\left(x_{0}\right)-z_{0}\right)+\frac{2}{3} C_{V} t^{3}\left|\partial_{x} V\left(x_{0}\right)-z_{0}\right| \psi(t)
\end{aligned}
$$

with $|\psi(t)| \leq 1$ on $\left[0, t_{V}\right]$. Recalling (6.14) and using the triangular inequality, we get for $t \in\left[0, t_{V}\right]$ that if $t_{V} \leq 3 /\left(8 C_{V}\right)$,

$$
\begin{aligned}
\left|x(t)-z_{0}\right|^{2} \geq \max \{ & \left|x_{0}-z_{0}\right|-t\left|y_{0}\right|-t^{2}\left|\partial_{x} V\left(x_{0}\right)-z_{0}\right|, \\
& t\left|y_{0}\right|-\left|x_{0}-z_{0}\right|-t^{2}\left|\partial_{x} V\left(x_{0}\right)-z_{0}\right|, \\
& \left.\frac{t^{2}}{4}\left|\partial_{x} V\left(x_{0}\right)-z_{0}\right|-\left|x_{0}-z_{0}\right|-t\left|y_{0}\right|\right\}
\end{aligned}
$$

Let us now take again $0<\theta<t_{V}$. We consider three cases:

1. If $\left|x_{0}-z_{0}\right| \geq \max \left\{\theta\left|y_{0}\right|, 18 \theta^{2}\left|\partial_{x} V\left(x_{0}\right)-z_{0}\right|\right\}$, we use the first expression in (6.23) and we get that

$$
\left|x(t)-z_{0}\right|^{2} \geq\left|x_{0}-z_{0}\right|\left(1-t / \theta-18 t^{2} / \theta^{2}\right) \geq \frac{1}{4}\left|x_{0}-z_{0}\right| \quad \text { on } \quad[0, \theta / 6] .
$$

2. If $\theta\left|y_{0}\right| \geq \max \left\{\left|x_{0}-z_{0}\right|, 18 \theta^{2}\left|\partial_{x} V\left(x_{0}\right)-z_{0}\right|\right\}$, we use the second expression in (6.23) and we get that

$$
\left|x(t)-z_{0}\right|^{2} \geq\left|y_{0}\right|\left(t-\theta-t^{2} /(18 \theta)\right) \geq \frac{\theta}{2}\left|y_{0}\right| \quad \text { on } \quad[2 \theta, 3 \theta] .
$$

3. If $18 \theta^{2}\left|\partial_{x} V\left(x_{0}\right)-z_{0}\right| \geq \max \left(\left|x_{0}-z_{0}\right|, \theta\left|y_{0}\right|\right)$, we use the third expression in (6.23) and we get that

$$
\begin{aligned}
& \left|x(t)-z_{0}\right|^{2} \geq\left|\partial_{x} V\left(x_{0}\right)-z_{0}\right|\left(\frac{1}{4} t^{2}-18 t \theta-18 \theta^{2}\right) \geq \frac{t_{V}^{2}}{16}\left|\partial_{x} V\left(x_{0}\right)-z_{0}\right| \text { on }\left[t_{V} / 2, t_{V}\right] . \\
& \text { if } 18 \theta^{2} \leq t_{V}^{2} / 16 .
\end{aligned}
$$

In all cases we get that there exists a positive constant $c_{V}^{\prime}$ only depending on $C_{V}$ such that

$$
\left|x(t)-z_{0}\right| \geq c_{V}^{\prime} \max \left\{\left|y_{0}\right|,\left|x_{0}-z_{0}\right|,\left|\partial_{x} V\left(x_{0}\right)-z_{0}\right|\right\}
$$

on an interval of length at least $\theta$. 
Conclusion From (6.18), (6.24) and using (6.14), we get that in all cases there exists an interval of length at least $\min \left(\theta, t_{V} / 2\right)$ on which

$$
\begin{aligned}
p_{0}\left(\exp (t \nu)\left(x_{0}, y_{0}, z_{0}\right)\right) & =\frac{\gamma}{2}\left|x(t)-z_{0}\right|^{2} \\
& \geq \frac{\gamma}{2}\left(\min \left(c_{V}^{\prime}, c_{V}\right) \max \left\{\left|y_{0}\right|,\left|x_{0}-z_{0}\right|,\left|\partial_{x} V\left(x_{0}\right)-z_{0}\right|\right\}\right)^{2} .
\end{aligned}
$$

Since $\left(x_{0}, y_{0}, z_{0}\right) \notin \pi_{x y z}(\mathcal{C}+B(0, \varepsilon))$ and using (6.13) we get that the dynamical condition (2.16) is fulfilled. The proof is complete.

\section{References}

[1] J. M. Bismut, The hypoelliptic Laplacian on the cotangent bundle, J. Amer. Math. Soc. 18(2005), 379-476.

[2] J. M. Bismut, G. Lebeau, The hypoelliptic Laplacian and Ray-Singer metrics, preprint (2006).

[3] N. Dencker, J. Sjöstrand, and M. Zworski, Pseudospectra for semiclassical pseudodifferential operators, Comm. Pure Appl. Math. 57 (2004), 384-415.

[4] M. Dimassi, J. Sjöstrand, Spectral asymptotics in the semi-classical limit, Cambridge University Press, 1999.

[5] J.P. Eckmann and M. Hairer, Non-equilibrium statistical mechanics of strongly anharmonic chains of oscillators, Comm. Math. Phys. 212 (2000), 105-164.

[6] J.P. Eckmann and M. Hairer, Spectral properties of hypoelliptic operators, Comm. Math. Phys. 235 (2003), 233-253.

[7] J.P. Eckmann, C.A. Pillet, and L. Rey-Bellet, Non-equilibrium statistical mechanics of anharmonic chains coupled to two heat baths at different temperatures, Comm. Math. Phys. 201 (1999), 657-697.

[8] G. Lebeau, Le bismutien, Séminaire équations aux dérivées partielles, Ecole Polytechnique 2004-05, I.1-I.15

[9] M. Hairer and J.C. Mattingly, Slow energy dissipation in systems of anharmonic oscillators, preprint, 29 p., 2007. 
[10] F. Hérau and F. Nier, Isotropic hypoellipticity and trend to equilibrium for the Fokker-Planck equation with high degree potential, Arch. Ration. Mech. Anal. 171 (2004), 151-218.

[11] F. Hérau, J. Sjöstrand, and C. Stolk, Semiclassical analysis for Kramers-FokkerPlanck type operators, Comm. Partial Differential Equations 30 (2005), 689-760.

[12] F. Hérau, M. Hitrik, and J. Sjöstrand, Tunnel effect for Kramers-Fokker-Planck type operators, Annales Henri Poincaré, to appear.

[13] F. Hérau Méthodes microlocales pour les équations cinétiques, mémoire d'habilitation à diriger des recherches, Reims, 2007.

[14] L. Hörmander, Symplectic classification of quadratic forms and general Mehler formulas, Math. Zeitschrift, 219 (1995), 413-449.

[15] V. N. Kolokoltsov, Semiclassical analysis for diffusions and stochastic processes, Springer LNM 1724, 2000.

[16] H. A. Kramers, Brownian motion in a field of force and the diffusion model of chemical reactions, Physica 7(4) (1940), 284-304.

[17] E. Nelson, Dynamical theories of Brownian motion, Princeton University Press, Princeton, N.J., 1967.

[18] B. Øksendal, Stochastic Differential equations, Springer, 2000

[19] A. Pazy, Semigroups of linear operators and applications to partial differential equations, Springer-Verlag, Berlin, second edition, 1983.

[20] J. Sjöstrand, Parametrices for pseudodifferential operators with multiple characteristics, Ark. Mat. 12 (1974), 85-130.

[21] J. Tailleur, S. Tanase-Nicola, J. Kurchan, Kramers equation and supersymmetry, J. Stat. Phys. 122 (2006), no. 4, 557-595.

[22] E. Witten, Supersymmetry and Morse theory. J. Differential Geom., 17 no. 4, 661-692, 1982.

[23] C. Villani, Hypocoercivity, to appear in Memoirs Amer. Math. Soc., available at http://www.umpa.ens-lyon.fr/ cvillani/Cedrif/pre.Hypoco.pdf, 2007. 